\title{
Coronary Artery Disease Associated Transcription Factor TCF21 Regulates Smooth Muscle Precursor Cells That Contribute to the Fibrous Cap
}

\author{
Sylvia T. Nurnberg ${ }^{1 \oplus}$, Karen Cheng ${ }^{1 \oplus}$, Azad Raiesdana ${ }^{1}$, Ramendra Kundu ${ }^{1}$, \\ Clint L. Miller ${ }^{1}$, Juyong B. Kim ${ }^{1}$, Komal Arora ${ }^{2}$, Ivan Carcamo-Oribe ${ }^{1}$, Yiqin Xiong ${ }^{1}$, \\ Nikhil Tellakula ${ }^{1}$, Vivek Nanda', Nikitha Murthy ${ }^{1}$, William A. Boisvert ${ }^{2}$, Ulf Hedin ${ }^{3}$, \\ Ljubica Perisic ${ }^{3}$, Silvia Aldi ${ }^{3}$, Lars Maegdefessel ${ }^{4}$, Milos Pjanic ${ }^{1}$, Gary K. Owens ${ }^{5}$, \\ Michelle D. Tallquist ${ }^{2}$, Thomas Quertermous ${ }^{1}{ }^{*}$ \\ 1 Department of Medicine, Cardiovascular Research Institute, Stanford University School of Medicine, \\ Stanford, California, United States of America, 2 Department of Medicine, University of Hawaii, Honolulu, \\ Hawaii, United States of America, 3 Department of Molecular Medicine and Surgery, Karolinska Institute, \\ Stockholm, Sweden, 4 Department of Medicine, Karolinska Institute, Stockholm, Sweden, 5 Department of \\ Molecular Physiology and Biological Physics, University of Virginia School of Medicine, Charlottesville, \\ Virginia, United States of America \\ - These authors contributed equally to this work. \\ * tomq1@stanford.edu
}

Citation: Nurnberg ST, Cheng K, Raiesdana A,
Kundu R, Miller CL, Kim JB, et al. (2015) Coronary Artery Disease Associated Transcription Factor TCF21 Regulates Smooth Muscle Precursor Cells That Contribute to the Fibrous Cap. PLoS Genet 11(5): e1005155. doi:10.1371/journal.pgen.1005155

Editor: Mark I. McCarthy, University of Oxford, UNITED KINGDOM

Received: November 13, 2014

Accepted: March 18, 2015

Published: May 28, 2015

Copyright: $\odot 2015$ Nurnberg et al. This is an open access article distributed under the terms of the Creative Commons Attribution License, which permits unrestricted use, distribution, and reproduction in any medium, provided the original author and source are credited.

Data Availability Statement: All data files are available from the GEO database (accession number GSE44461) and may be accessed at: http://www. ncbi.nlm.nih.gov/geo/query/acc.cgi?acc=GSE44461.

Funding: This work was supported by NIH grants HL103635 (TQ), HL109512 (TQ), HL057353 (GKO), HL074257 (MDT), HL81863 (WAB) and a grant from the Leducq Foundation (TQ). The BiKE project was supported by funds from the Swedish Heart and Lung Foundation, the Swedish Research Council (K200965X-2233-01-3, K2013-65X-06816-30-4 and 3492007-8703), Uppdrag Besegra Stroke (P581/2011-

\section{Abstract}

Recent genome wide association studies have identified a number of genes that contribute to the risk for coronary heart disease. One such gene, TCF21, encodes a basic-helix-loophelix transcription factor believed to serve a critical role in the development of epicardial progenitor cells that give rise to coronary artery smooth muscle cells (SMC) and cardiac fibroblasts. Using reporter gene and immunolocalization studies with mouse and human tissues we have found that vascular TCF21 expression in the adult is restricted primarily to adventitial cells associated with coronary arteries and also medial SMC in the proximal aorta of mouse. Genome wide RNA-Seq studies in human coronary artery SMC (HCASMC) with siRNA knockdown found a number of putative TCF21 downstream pathways identified by enrichment of terms related to CAD, including "vascular disease," "disorder of artery," and "occlusion of artery," as well as disease-related cellular functions including "cellular movement" and "cellular growth and proliferation." In vitro studies in HCASMC demonstrated that TCF21 expression promotes proliferation and migration and inhibits SMC lineage marker expression. Detailed in situ expression studies with reporter gene and lineage tracing revealed that vascular wall cells expressing $T c f 21$ before disease initiation migrate into vascular lesions of $A p o E^{-1-}$ and $L d l r^{-1-}$ mice. While Tcf21 lineage traced cells are distributed throughout the early lesions, in mature lesions they contribute to the formation of a subcapsular layer of cells, and others become associated with the fibrous cap. The lineage traced fibrous cap cells activate expression of SMC markers and growth factor receptor genes. Taken together, these data suggest that TCF21 may have a role regulating the 
123), the Strategic Cardiovascular Programs of Karolinska Institute and Stockholm County Council, the Stockholm County Council (ALF2011-0260 and ALF-2011-0279). The funders had no role in study design, data collection and analysis, decision to publish, or preparation of the manuscript.

Competing Interests: The authors have declared that no competing interests exist. differentiation state of SMC precursor cells that migrate into vascular lesions and contribute to the fibrous cap and more broadly, in view of the association of this gene with human $C A D$, provide evidence that these processes may be a mechanism for CAD risk attributable to the vascular wall.

\section{Author Summary}

Coronary artery disease (CAD) is responsible for the majority of deaths in the Western world, and is due in part to environmental and metabolic factors. However, half of the risk for developing heart disease is genetically predetermined. Genome-wide association studies in human populations have identified over 100 sites in the genome that appear to be associated with $\mathrm{CAD}$, however, the mechanisms by which variation in these regions are responsible for predisposition to CAD remain largely unknown. We have begun to study a gene that contributes to CAD risk, the TCF21 gene. Through genomic studies we show that this gene is involved in processes related to alterations in vascular gene expression, and in particular those related to the smooth muscle cell biology. With cell culture models, we show that TCF21 regulates the differentiation state of this cell type, which is believed critical for vascular disease. Using mouse genetic models of atherosclerotic vascular disease we provide evidence that this gene is expressed in precursor cells that migrate into the disease lesions and contribute to the formation of the fibrous cap that is believed to stabilize these lesions and prevent heart attacks.

\section{Introduction}

Atherosclerotic coronary heart disease (CAD) continues to be the dominant medical problem in the Western world and is growing in minority groups in this country and in developing populations [1-4]. Despite extensive investigation of epidemiological and cellular features of the disease, there are still fundamental questions related to the mechanism of etiology that remain to be answered. For instance, the resident SMC that constitute the majority of the native vessel wall undergo dramatic phenotypic changes in the disease setting, with the loss of SMC gene expression in the medial vessel layer and appearance of a new layer of SMC marker expressing cells in a "fibrous cap" that covers the lipid core of the plaque. While the risk of plaque rupture appears to be inversely correlated with the number of SMC-like cells in the fibrous cap, there is very little understanding of their in vivo origin, and the molecular pathways that regulate their expansion and terminal phenotype determination [5]

Significant new insights into the fundamental cellular processes that drive CAD and other complex human diseases have recently been achieved through genome wide association (GWA) studies employing large cohorts of patients and healthy controls. These studies have provided the first incontrovertible identification of genes and disease pathways that affect risk for many complex human diseases (www.genome.gov/gwastudies), including CAD. A recent GWA meta-analysis has identified 46 loci that associate with CAD at the genome-wide significance level of $5 \times 10^{-8}$, and another 104 independent variants that associate at a false discovery rate less than 0.05 [6]. Of these CAD associated loci, more than two-thirds act independently of traditional risk factors, and are likely to modulate risk of CAD through regulation of cellular processes in the blood vessel wall. Further study of this latter group of genes is expected to provide insights into novel atherosclerosis pathways. 
One CAD associated gene that has not been linked to known environmental or metabolic risk factors is the basic-helix-loop-helix transcription factor TCF21 (capsulin, POD1, epicardin) [6-8]. This gene was initially cloned in four independent laboratories and shown to mark expression of mesodermal cells that give rise to kidney, lung, spleen, gonads, and a restricted group of cranial muscle cells [9-16]. Recent studies have shown that Tcf21 regulates fundamental cell fate decisions in the developing epicardium, serving as a determining factor for divergence between coronary vascular smooth muscle cell and cardiac fibroblast lineages $[17,18]$. In this setting, Tcf21 is downregulated in cells that are fated to become differentiated coronary SMC and has sustained expression in cells that become interstitial and adventitial fibroblasts [18]. However, its role in molecular and cellular processes related to CAD has not been investigated.

To examine the possible role of TCF21 in coronary artery disease, we assessed its expression pattern in normal and diseased tissues, investigated its role in fundamental cellular processes, and employed next generation sequencing based methods in SMC to identify genes and pathways regulated by TCF21 at the transcriptional level. We have also identified the temporal and cell-specific expression of $T c f 21$ in reporter gene and lineage tracing mouse models to better understand how this gene might regulate vascular wall cellular processes in the setting of atherosclerotic disease.

\section{Results}

\section{TCF21 is expressed in human SMC and other mesodermal lineages in vitro and in human and mouse coronary arteries}

To better understand the expression pattern of TCF21 in adult tissues, we studied expression in a number of adult human cells by quantitative real-time polymerase chain reaction (qPCR) (S1A Fig). TCF21 was expressed in human coronary artery smooth muscle cells (HCASMC), but not aortic smooth muscle cells (HAoSMC) or endothelial cells isolated from coronary (HCAEC) or aortic vessels (HAoEC). Because of the reported similarity between pericytes, which have been developmentally linked to Tcf21 [10], and mesenchymal stem cells (MSC) [19], we investigated expression in MSC derived from bone marrow (bmMSC), embryonicstem cells (eMSC) and induced pluripotent stem cells (iMSC). Results showed that all of these cells expressed TCF21. Cardiac fibroblasts (HCF) and adventitial aortic fibroblasts (HAoAF) were also found to express TCF21.

Immunocytochemistry of cultured HCASMC revealed nuclear expression of TCF2 1 in cells that were also expressing the SMC marker ACTA2, although a number of prominent cells expressing high levels of ACTA2 appeared to have low-level expression of TCF21 (S1B Fig). In situ hybridization of mouse and human coronary artery specimens with species-specific cRNA probes revealed prominent labeling of adventitial cells with no staining of medial SMC or intimal endothelial cells (S1C Fig).

In preparation for studies in mouse genetic disease models, we investigated $T c f 21$ expression in normal adult mice with Xgal staining of tissues containing a lac $Z$ reporter gene integrated into the murine Tcf 21 locus, and compared this staining with that seen in C57BL/6 mice $[12,20]$. As with in situ hybridization studies in mouse and human tissues, results showed staining of cells in the adventitia of both the coronary arteries and aorta, with primary localization in those adventitial cells juxtaposed to the vascular media (S1D Fig). Unique to the mouse, there was a patchy distribution of lacZ expressing cells in the proximal aortic wall (S1E Fig). When $\beta$-galactosidase activity was pseudocolored green and merged with the Acta2 immunoreactivity (red) there appeared to be minimal overlap in either the coronary arteries or aorta. 


\section{Genomic studies identify disease and developmental pathways downstream of TCF21}

To provide functional insights into the role of TCF21 in adult SMC in the disease setting, we employed whole transcriptome RNA sequencing (RNA-Seq) studies on cells transfected with either non-silencing control siRNA (siCTRL) or silencing TCF21 siRNA (siTCF21). Quantitative RT-PCR of HCASMC transfected with siTCF21 compared to siCTRL showed a significant decrease in mRNA levels for TCF21 ( $0.99 \pm 0.02$ control vs. $0.25 \pm 0.02$ siTCF21, $P<0.0001)$

(S2A Fig), and quantitation of western blots of protein extracts showed a similar reduction of TCF21 protein levels to $26 \%$ of baseline (S2B Fig).

We employed two commonly used algorithms, DESeq and edgeR, to identify genes that were differentially expressed (FDR $\leq 0.05$ ) between the HCASMC treated with siTCF21 or siCTRL reagents $[21,22]$. To eliminate outliers that might be identified by each analysis, we intersected the 466 genes obtained with DESeq and 430 genes found with edgeR to obtain 380 genes that were common to the two approaches [23]. The intersected genelist was mapped to the Ingenuity IPA Knowledge Base, generating algorithmically computed pathways or networks that were then investigated for over-representation in disease and biological function categories. "Connective Tissue Development and Function, Tissue Morphology, Cardiovascular Disease" was identified as the top network. "Cardiovascular Disease" was ranked third in the disease category, with 20 subcategories including "Vascular disease" $(\mathrm{Q}=3.26 \mathrm{E}-17$, righttailed Fisher's Exact Test, Benjamini-Hochberg corrected), "Disorder of artery" (Q = 4.04E$12)$, and "Occlusion of artery" $(\mathrm{Q}=2.03 \mathrm{E}-14)$ as the top three functional groups (Tables 1 and S1). "Cardiovascular System Development and Function" ( $Q=1.88 \mathrm{E}-16-5.30 \mathrm{E}-04)$ was the most highly ranked in the "Physiological System Development and Function" category, with a number of subcategories that were related to embryonic development, including "Development of the cardiovascular system" ( $\mathrm{Q}=1.88 \mathrm{E}-16)$, "Angiogenesis" $(\mathrm{Q}=4.58 \mathrm{E}-14)$, and "Development of blood vessel" ( $\mathrm{Q}=4.58 \mathrm{E}-14)$ being the top three functional groups (Tables 1 and S2). Among the Molecular and Cellular Functions category of traits, the top three functional groups were "Cellular movement" $(\mathrm{Q}=4.23 \mathrm{E}-23)$, followed by "Cellular growth and proliferation" $(\mathrm{Q}=4.23 \mathrm{E}-23)$, and "Cell death and survival" $(\mathrm{Q}=1.06 \mathrm{E}-18)$ (Tables 1 and $\mathrm{S} 3$ ).

Taking the first subcategory in the "Cardiovascular Disease" category, "Vascular disease," we used well-curated molecular interactions in the IPA Knowledge Base to build a gene network. The initial group included 73 genes, and 18 additional genes were added with the "build" function in IPA, this group functionally related with the "connect" function, and with elimination of non-connected nodes resulted in a final TCF21 Vascular Disease Network containing 83 genes (Fig 1). Also, content was added to the network with annotation from Gene Ontology classifications ( $\mathrm{S} 3 \mathrm{Fig}$ ). One of the strongest signals in the network was the upregulation of matrix components with TCF21 knockdown, including a large number of collagens, SPARC, LOX, vitronectin $(V T N)$, and components of the matrix that are known to affect vascular cell functions, including thrombospondin 1 (THBS1) and fibrillin 1 (FBN1), as well as vascular cell surface proteins that interact with the matrix including alpha $\mathrm{V}$ integrin (ITGAV). Although the matrix degrading enzymes MMP7, MMP10 and MMP25 are upregulated with TCF21 knockdown, the downregulation of the much more highly expressed $M M P 1$ and $M M P 3$ combined with the upregulation of MMP inhibitor TIMP3 are consistent with an anti-matrix overall profile of a mobile cell. Also upregulated are SMC markers such as smooth muscle actin (ACTA2) and myosin light chain kinase (MYLK). Among the genes downregulated with TCF21 knockdown are those involved in vascular development, including neuropilin 1 (NRP1), VEGF receptors II (KDR), angiopoietin 1 (ANGPT1), TGF $\beta$ receptor 2 (TGFBR2), and semaphorin 3D (SEMA3D), and cytokines and chemokines such as IL1A, IL1B, CXCL2 and CXCL3. Taken 
Table 1. Functional annotation derived from analysis of differentially expressed genes in siTCF21 RNA-Seq experiments.

\begin{tabular}{|c|c|}
\hline \multicolumn{2}{|c|}{ CARDIOVASCULAR DISEASE } \\
\hline Functional annotation & Q-Value \\
\hline Vascular disease & $3.26 \mathrm{E}-17$ \\
\hline Disorder of artery & 8.47E-17 \\
\hline Occlusion of artery & $4.04 \mathrm{E}-12$ \\
\hline Aneurysm & 2.99E-09 \\
\hline Vascular lesion & $6.68 \mathrm{E}-08$ \\
\hline Peripheral arterial occlusive disease & $1.23 \mathrm{E}-07$ \\
\hline Aortic aneurysm & $2.53 \mathrm{E}-07$ \\
\hline Aortic disorder & $7.58 \mathrm{E}-07$ \\
\hline Arteriosclerosis & 2.15E-06 \\
\hline Atherosclerosis & 4.63E-06 \\
\hline Arterial dissection & $2.31 \mathrm{E}-05$ \\
\hline Cardiomyopathy & 3.34E-05 \\
\hline Advanced stage peripheral arterial occlusive disease & 4.35E-05 \\
\hline Mid-stage peripheral arterial occlusive disease & 7.57E-05 \\
\hline Infarction & 8.59E-05 \\
\hline \multicolumn{2}{|c|}{ CARDIOVASCULAR SYSTEM DVLP \& FUNCTION } \\
\hline Functional annotation & Q-Value \\
\hline Development of cardiovascular system & $1.88 \mathrm{E}-16$ \\
\hline Angiogenesis & $4.58 \mathrm{E}-14$ \\
\hline Development of blood vessel & $4.58 \mathrm{E}-14$ \\
\hline Morphology of cardiovascular system & $2.20 \mathrm{E}-13$ \\
\hline Vasculogenesis & $6.32 \mathrm{E}-13$ \\
\hline Abnormal morphology of cardiovascular system & 8.11E-11 \\
\hline Cell movement of endothelial cells & 4.37E-10 \\
\hline Migration of endothelial cells & 5.11E-09 \\
\hline Adhesion of endothelial cell lines & $2.03 \mathrm{E}-08$ \\
\hline Endothelial cell development & $3.82 \mathrm{E}-08$ \\
\hline Neovascularization & 1.67E-07 \\
\hline Angiogenesis of tumor & 4.63E-07 \\
\hline Angiogenesis of extraembryonic tissue & $5.39 \mathrm{E}-07$ \\
\hline Proliferation of endothelial cells & $1.68 \mathrm{E}-08$ \\
\hline Morphology of blood vessel & $1.71 \mathrm{E}-06$ \\
\hline Abnormal morphology of blood vessel & 2.09E-06 \\
\hline \multicolumn{2}{|c|}{ MOLECULAR AND CELLULAR FUNCTIONS } \\
\hline Functional annotation & Q-Value \\
\hline Cellular movement & $4.23 E-23$ \\
\hline Cellular growth and proliferation & $4.23 \mathrm{E}-23$ \\
\hline Cell death and survival & $1.06 \mathrm{E}-18$ \\
\hline Cellular development & $2.76 \mathrm{E}-14$ \\
\hline Cellular function and maintenance & 2.10E-09 \\
\hline
\end{tabular}

doi:10.1371/journal.pgen.1005155.t001 


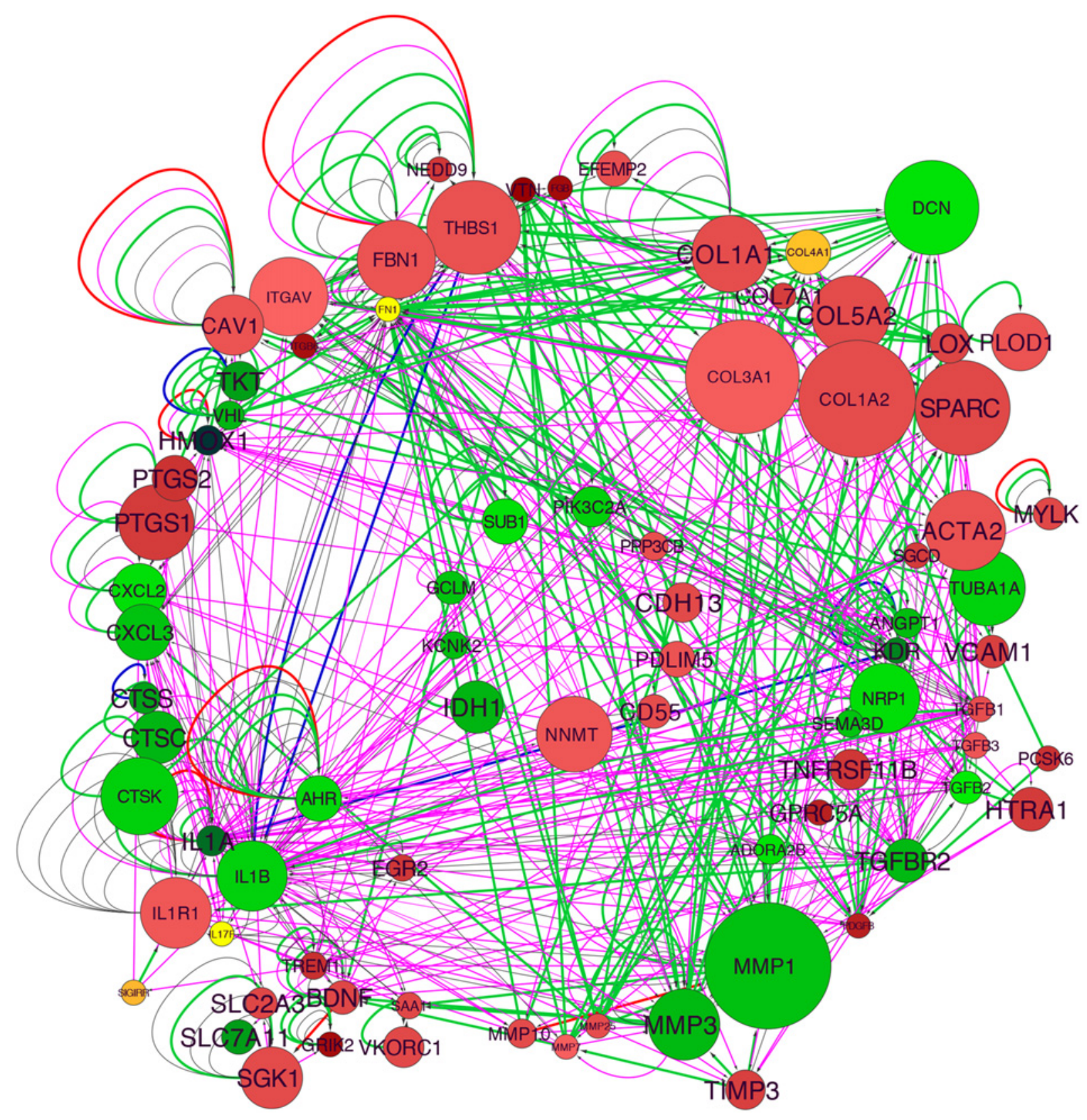

Fig 1. TCF21 Vascular Disease Network built with differential gene expression data from siTCF21 RNA-Seq studies in HCASMC. Interaction of the network nodes identified through enrichment of differentially expressed genes in functionally annotated categories was visualized with Cytoscape. Node color was mapped to log fold change with green representing genes that are downregulated along with TCF21 and red representing genes that are upregulated, node size was mapped to absolute expression value in control cells, and font size to enrichment $Q$-value. Edges contain arrows to indicate the direction of interaction and are colored to distinguish types of interactions. Green edges represent functional interaction (protein-protein binding, protein modification, molecular cleavage, phosphorylation, and protein-DNA interactions); magenta edges represent gene expression (expression and transcription) relationships; red edges represent activation; and blue edges inhibition.

doi:10.1371/journal.pgen.1005155.g001

together these data suggest that TCF21 positive cells express low levels of many matrix proteins while expressing high levels of matrix degrading enzymes, consistent with a pro-migratory phenotype, express low levels of SMC lineage markers, and are highly pro-angiogenic and proinflammatory, a phenotype quite different from vascular wall SMC. 


\section{TCF21 directly regulates cell fate decisions in cultured SMC}

To investigate a functional role for TCF21 in fundamental cell fate decisions in vascular SMC, we employed lentiviral shRNA as well as siRNA mediated knockdown and lentiviral overexpression studies. Control lentiviral vectors (pWPI) and lentiviral over-expression vectors (pWPI-TCF21), and control (pLVTHM) and lentiviral shRNA mediated knockdown vectors (pLVTHM-shTCF21) were used to transduce primary cultured HCASMC. pWPI-TCF21 increased TCF21 mRNA levels (1.0 \pm 0.04 pWPI vs. 32.5 \pm 0.02 pWPI-TCF21, $\mathrm{P}<0.0001)$, and

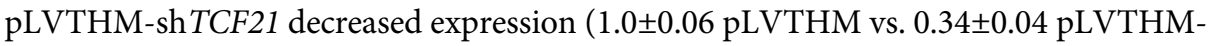
shTCF21 2, P < 0.001) (S4A Fig). Western blot of protein extracts from HCASMC that were transduced with over-expression and knockdown lentiviruses showed a 4.5 -fold increase, and reduction of TCF 21 protein levels to $8 \%$ (shTCF21 1, shTCF21 2) of baseline respectively (S4B Fig).

To assess the effect of TCF21 on rate of cell division in vitro, proliferation rates of pools of stably transduced and non-transduced cells were measured over time, where increased or decreased proliferation rates in transduced cells would lead to a change in percentages of this GFP positive cell population as compared to the non-transduced, GFP negative population. Flow cytometry analysis of stable overexpression of TCF21 in HCASMC showed an increase in TCF21 overexpressing cells from 48 to 82 percent of the culture within 28 days (S5A Fig). In the same time period the percentage of cells with stable knockdown of TCF21 decreased from 53 to 16 for construct 1 (shTCF21 1 vs. empty control virus pLVTHM $P<0.0001$ ) and from 59 to 2 percent for construct 2 (shTCF21 2 vs. empty control virus pLVTHM $P<0.0001$ ) (S5B Fig). The percentage of GFP positive cells did not change when transduced with the empty vector control construct. To provide additional support for these findings we conducted EdU (5-ethynyl-2'-deoxyuridine) labeling proliferation assays. HCASMC transduced with the control, overexpression or knockdown lentiviruses were treated with EdU and imaged for nuclear fluorescence. These data showed an increase in the percentage of TCF21 overexpressing cells compared to DAPI stained cells $(33.1 \% \pm 2.3$ control vs. $51 \% \pm 4.1$ overexpressing cells, $P<0.001)$, and significant decreased percentage of dividing cells transduced with knockdown lentiviruses $(43.4 \% \pm 4.4$ control vs. $30.8 \% \pm 2.4$ knockdown cells, $P<0.01)$ (Fig $2 \mathrm{~A}$ and $2 \mathrm{~B}$ ). These data suggest that TCF21 positively affects SMC proliferation.

We also evaluated the effect of TCF21 on apoptosis and migration in cultured HCASMC. Apoptosis was evaluated in HCASMC transfected with siControl or siTCF21 RNAs and serum starved for 48 hrs. By caspase assay there was a significant decrease in apoptosis of cells transfected with the siTCF21 reagent $(41,824 \pm 1872$ vs. $18,837 \pm 1302$ for siCTRL compared to siTCF21, $P<0.0001$ ) (Fig 2C). For migration, HCASMC transduced with TCF21 overexpression lentivirus were evaluated with a gap closure assay. Here, TCF21 overexpressing cells covered a significantly larger surface area after 12 hours of incubation $(27.8 \pm 2.7$ vs. $15.8 \pm 1.16$ control cells, $P<0.001$ ) (Fig 2D). With an average doubling time of 71 hours for TCF21 overexpressing HCASMC this effect could not be due to the pro-proliferative effect of TCF21.

Given that TCF21 is downregulated in epicardial cells as they differentiate into coronary SMC, and a number of SMC lineage marker genes were upregulated in the siTCF21 RNA-Seq study, including ACTA2 and MYLK, we specifically investigated the expression of SMC marker genes in additional siRNA knockdown experiments. These studies showed that ACTA2, TAGLN and MYH11 transcript levels were significantly upregulated after 70\% TCF21 knockdown $(0.96 \pm 0.023$ vs. $1.38 \pm 0.16, P<0.05,1.0 \pm 0.03$ vs. $2.59 \pm 0.27, P<0.01$, and $1.05 \pm 0.09$ vs. $2.67 \pm 0.51, P<0.05$, respectively) (Fig $2 \mathrm{E}$ ), suggesting their direct suppression by TCF2 1 at the transcriptional level. 
A

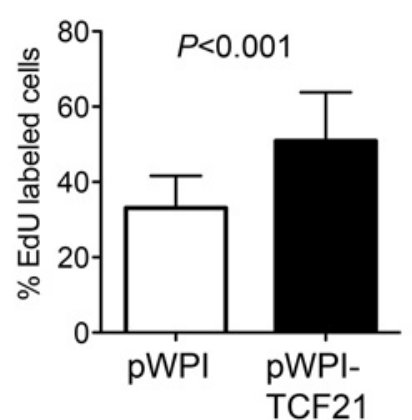

B

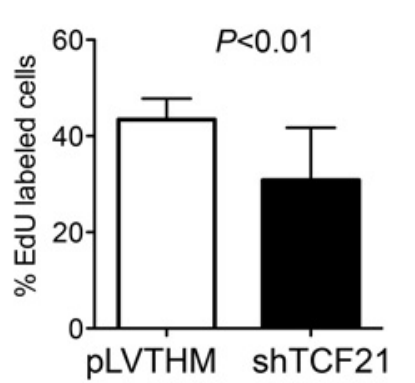

C

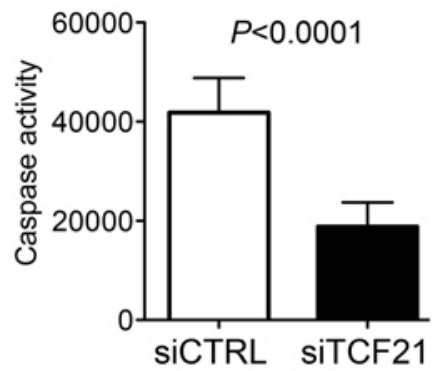

D

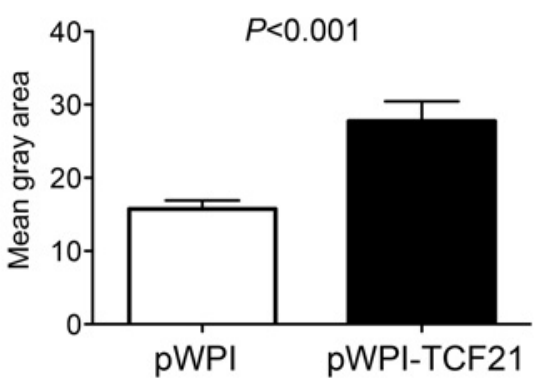

E

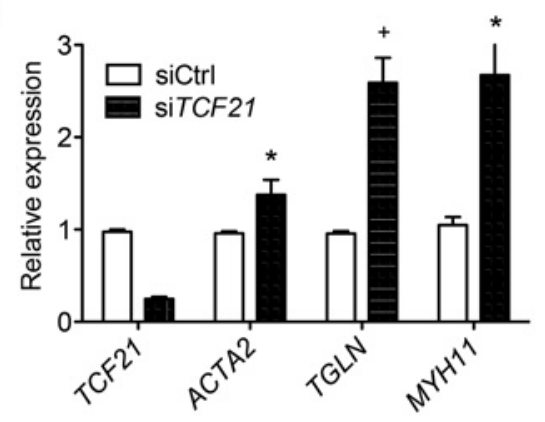

$\mathrm{F}$

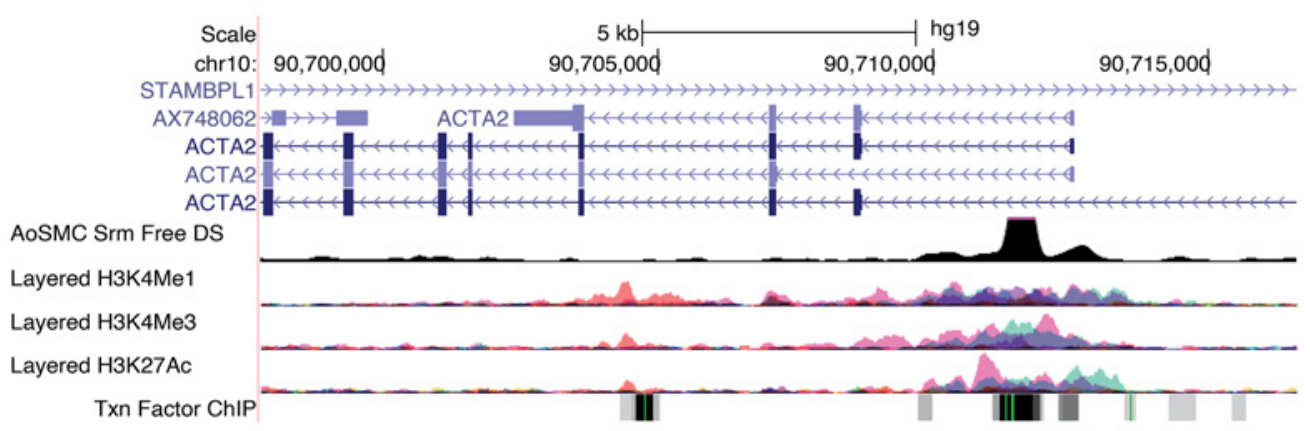

G

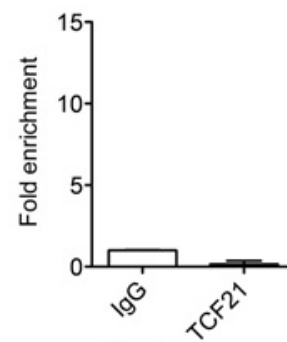

Control

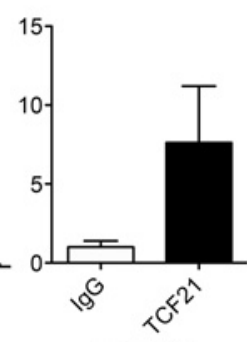

ACTA2
$\mathrm{H}$

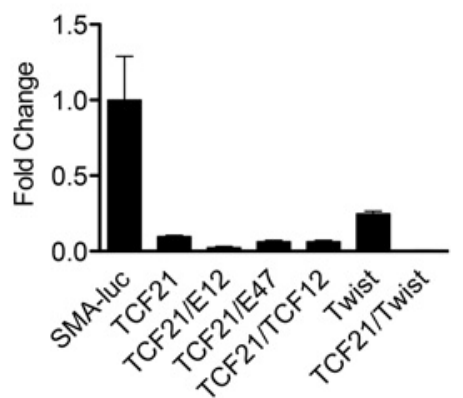

I

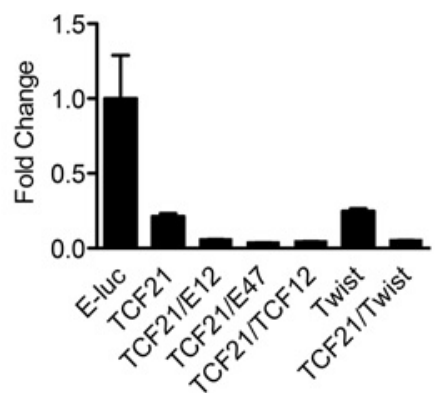

Fig 2. TCF21 regulates basic cellular functions in vascular smooth muscle cells in vitro. A) HCASMC transduced with TCF21 overexpressing lentivirus (pWPI-TCF21) or empty lentivirus (pWPI empty) were labeled with the thymidine analogue 5-ethynyl-2'-deoxyuridine (EdU), which was visualized with a fluorescent azide, allowing identification and quantification of proliferating cells with immunofluorescence microscopy, reported here as a percent with baseline being all DAPI positive cells. HCASMC showed an increase in the percentage of TCF21 overexpressing cells compared to DAPI stained cells $(33.1 \% \pm 2.3$ control vs. $51 \% \pm 4.1$ overexpressing cells, $P<0.001)$. B) HCASMC transduced with knockdown lentiviruses showed a decreased percentage of dividing cells $(43.4 \% \pm 4.4$ control vs. $30.8 \% \pm 2.4$ knockdown cells, $P<0.01)$. C) siTCF21 produced a decrease in apoptosis in HCASMC as measured by a caspase activity assay $(41,824 \pm 1872$ vs. $18,837 \pm 1302, P<0.0001)$. D) TCF21 regulation of HCASMC migration was evaluated with a gap closure assay. TCF21 overexpressing cells transduced with pWPI-TCF21 lentivirus covered a significantly larger surface area after 12 hours of study compared to cells transduced with the empty pWPI vector $(27.8 \pm 2.7$ vs. $15.8 \pm 1.16, P<0.001)$. E) HCASMC treated with siTCF21 compared to siCTRL showed significantly 
increased expression of ACTA2, TAGLN, and MYH11 SMC marker genes $(P<0.05 / P<0.01 / P<0.05$ respectively). F) The $A C T A 2$ locus as visualized on the University of California Santa Cruz genome browser. Data provided here reveals evidence for a likely enhancer region in the first intron, as indicated by DNase hypersensitivity measured in human aortic SMC, and histone modification data showing enrichment of H3K27Ac and H3K4me1 at this same site, as well as clustering of a number of transcription factor binding sites. G) Chromatin immunoprecipitation for TCF21 binding to the enhancer region of the ACTA2 locus by ChIP-qPCR $(P<0.05)$. H) Dual luciferase assays in rat aortic smooth muscle cells with a reporter construct containing the human $A C T A 2$ promoter and first intron (SMA-luc). A TCF21 expression construct was transfected with human TCF3 (E12), TCF3 (E47), TCF12, and Twist1 murine expression vectors, showing specific suppression of transcription of the SMA-luc reporter ( $1.0 \pm 0.01$ vs. $0.1 \pm 0.009, P<0.01$ for TCF21 alone). I) Similar dual luciferase assays using a $3 \mathrm{E}$-box containing minimal promoter construct (E-luc) based on the nucleotide sequence of the first intron ( $\mathrm{n}=3,3$ replicates), again showing TCF21 mediated suppression of transcription ( $1.0 \pm 0.29$ vs. $0.21 \pm 0.02, P<0.01$ for TCF21 alone).

doi:10.1371/journal.pgen.1005155.g002

To investigate whether TCF21 directly binds and regulates SMC marker genes, a series of studies were conducted with the ACTA2 gene as a representative SMC marker. It is well known that the regulatory regions of the $A C T A 2$ gene are localized in the upstream promoter and first intronic regions of the gene [24], and this information in conjunction with available ENCODE histone modification data, ENCODE DNase hypersensitivity data from human aortic smooth muscle cells, and localization of E-box binding motif sequences provided the approach for choosing a reporter construct for these studies (Fig 2F). Chromatin immunoprecipitation was employed to verify the in vivo binding of TCF 21 to a region of the first intron enhancer identified through this analysis (Fig $2 \mathrm{G}$ ). The luciferase reporter gene construct employed for these studies contained the human ACTA2 promoter and first intron. Obligate heterodimer partners of TCF21, TCF3 and TCF12, were included in these experiments to facilitate transcriptional activation [25]. Also, based on the high degree of conservation of sequence in the bHLH domain between TCF21 and Twist, and the similar inhibitory developmental actions of this related transcription factor, an expression construct for Twist was included to investigate possible interactions with TCF21 in the context of ACTA2 reporter gene expression [26]. When this reporter was transfected into rat aortic smooth muscle cells (RASMC) in conjunction with a TCF21 expression construct, it was found to decrease expression ( $1.0 \pm 0.01$ vs. $0.1 \pm 0.009$, $P<0.05)$, and even greater reductions were observed when the TCF21 expression vector was combined with another vector expressing one of the class II bHLH factors TCF3 (protein isoforms E12 or E47) (Fig 2H). TCF21 was also shown to inhibit expression of a minimal luciferase reporter containing 3 E-box sequences based on the known TCF12 motif (CAGCTG) in conjunction with a minimal promoter. There was significant repression with TCF21 expression alone $(1.0 \pm 0.29$ vs. $0.21 \pm 0.02, P<0.05)$, and additional reductions were noted when bHLH class II factors were included (Fig 2I). For both constructs, the developmental factor Twist produced a modest increase in TCF21 transrepression.

\section{TCF21 expressing cells are found in atherosclerotic lesions and associate with the fibrous cap}

To investigate disease-related expression of $T c f 21$ in the vascular wall, we performed timecourse studies in $T c f 21^{l a c Z /+}, A p o E^{-/-}$hyperlipidemic atherosclerotic mice, and compared Xgal staining in these tissues with that observed in negative control $A p o E^{-/-}$animals. After four weeks of high fat diet (HFD) there were no $\beta$-galactosidase expressing cells in the forming lesions, although there was apparent clustering of $\beta$-galactosidase positive cells on the luminal side of the media immediately below the areas where lesions had initiated growth (Fig 3). By eight weeks of HFD, lesions in most animals showed abundant $\beta$-galactosidase positive cells accumulating toward the luminal side of the atheromatous lesion, with streaks extending from media through the lesion toward the fibrous cap. $\beta$-galactosidase positive cells observed in the lesion appeared to be aligned orthogonal to the axis of orientation for cells in the media, suggestive of migratory behavior. By twelve weeks of HFD feeding there was extensive staining in 

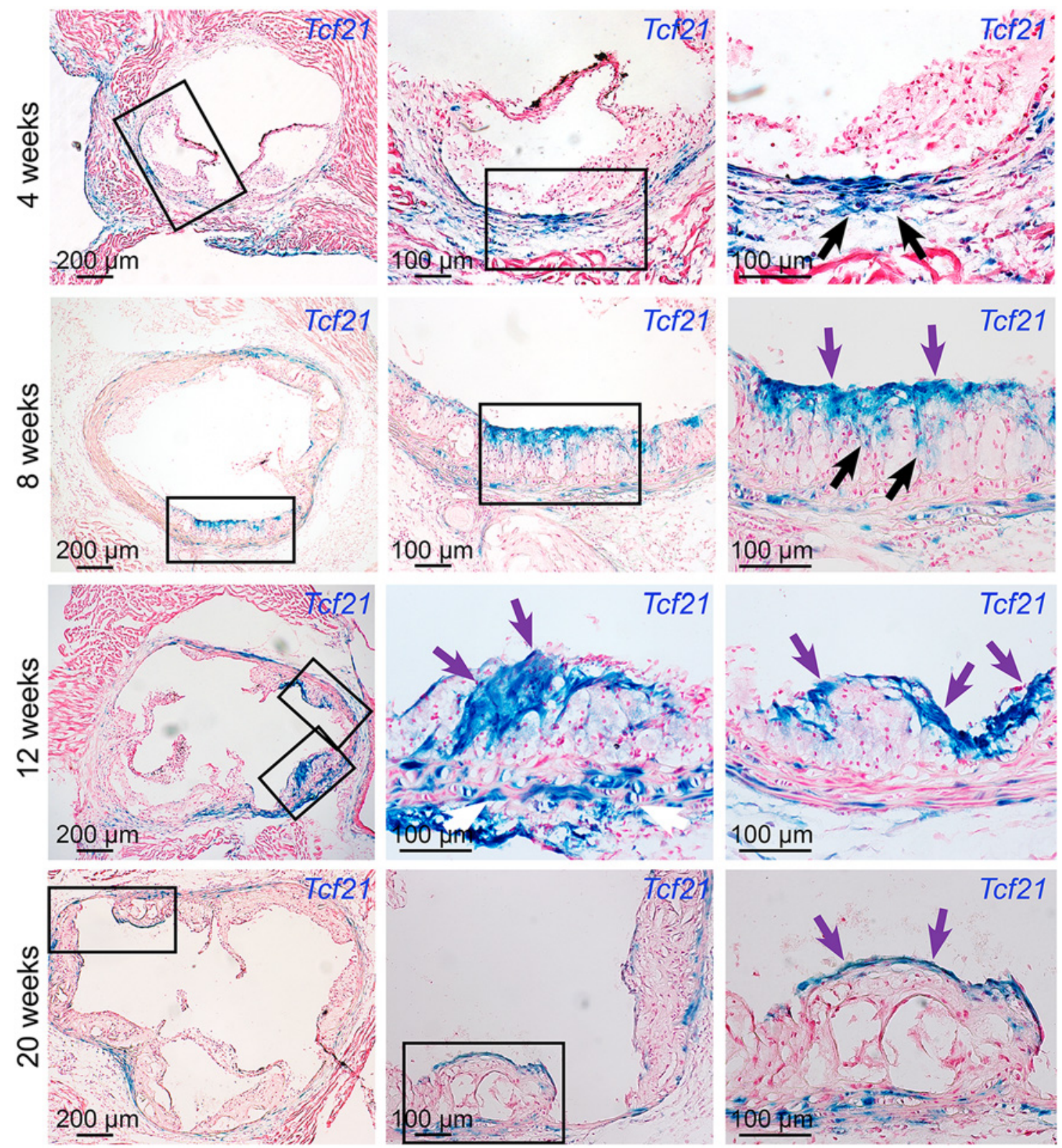

Fig 3. Tcf21 expressing cells are found in atherosclerotic lesions. Tcf2 $1^{1 a c Z /+}, A p o E^{-/-}$mice were fed HFD from 4 weeks of age for $4,8,12$, or 20 weeks, proximal aortic tissues were harvested, and Tcf21 gene expression evaluated by Xgal staining to visualize $\beta$-galactosidase activity, and sections were counterstained with nuclear fast red to visualize disease lesion architecture. For each timepoint, boxes in low-power images at left indicate regions examined at high power in panels to the right. At 4 weeks of HFD there was no expression in the lesions although clusters of lac $Z$ expressing cells identified in the media in regions below the disease lesions (arrows). At the 8-week timepoint cells with $\beta$-galactosidase activity were seen extending from the media to the luminal surface of the lesion (black arrows) in the vicinity of the forming fibrous cap (purple arrows). By 12 weeks of HFD there was extensive labeling of cells in lesions, with the appearance of $\beta$-galactosidase positive cells in the vicinity of the fibrous cap (purple arrows). Also, there was extensive staining of cells in areas of disrupted medial structure (white arrows). After 20 weeks of HFD, Tcf21-expressing cells had decreased in the lesions but formed a narrow band of cells associated with the fibrous cap (purple arrows).

doi:10.1371/journal.pgen.1005155.g003

lesions of most animals, with the $\beta$-galactosidase positive cells in the vicinity of the forming fibrous cap, or in a cap-like structure that was situated medial to and extended below the actual fibrous cap (Figs 3 and 4). This time interval from 4 to 12 weeks was associated with an apparent decrease in the number of medial cells expressing SMC markers and a disruption of the 

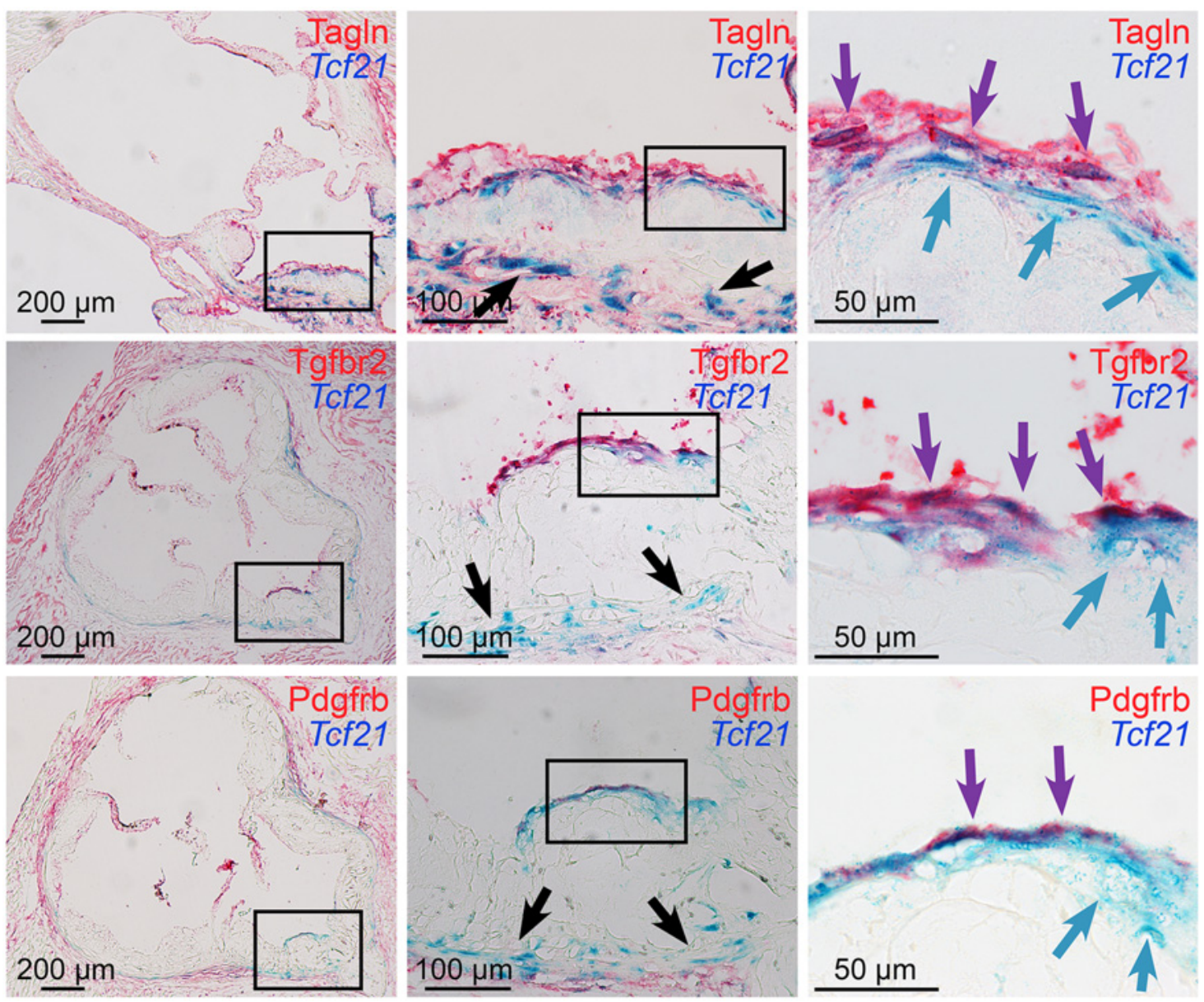

Fig 4. Tcf21 expressing cells associate with the fibrous cap. Xgal stained lesions in $T c f 21^{1 a c Z /+}, A p o E^{/-}$mice fed HFD for 20 weeks were evaluated by immunohistochemistry for expression of fibrous cap markers. Tcf21 reporter expressing cells (blue indicator) were identified in the media and adventitia (black arrows, middle panels), and in association with the fibrous cap (blue arrows, right panels). $\beta$-galactosidase negative cells in the luminal aspect of the fibrous cap stained positive for Tagln, as well as growth factor receptors Tgfbr2 and Pdgfrb (red indicator). In addition, in the region of the fibrous cap, cells were identified that showed staining for Tcf21 expression as well as Tagln and growth factor receptors (purple arrows).

doi:10.1371/journal.pgen.1005155.g004

lamellar medial structure. Tcf21 expressing cells in the media at 8 to 12 weeks were disorganized and had lost their characteristic lamellar structure. By 20 weeks, almost all of the $T c f 21$ expressing cells in the lesions were associated with the fibrous cap or a subcapsular structure (Fig 3).

To determine whether results in the ApoE model were consistent with TCF21 expression in the diseased human coronary vessel wall, immunostaining of human coronary artery tissues was performed with antibodies for TCF21 and ACTA2. In those vessels with only modest disease there was adventitial TCF21 staining with some staining of the minimal neointima, but no TCF21 staining in the media, which was positive for ACTA2 (S6A-S6C Fig). In vessels with significant disease there was robust TCF21 staining in the adventitia and neointima and some patchy staining in the fibrous cap, but very little staining in the media (S6D and S6E Fig). ACTA2 staining was prominent in the fibrous cap and considerably weaker staining in the media. These studies confirm what was observed in the mouse diseased vasculature.

To assign TCF21 expressing cells to a known cellular lineage we evaluated combined lacZ expression with immunohistochemistry staining for well-known lineage markers for different 
vascular cell types. Specifically, we worked to identify co-staining for murine adventitial and vascular lesion lineage markers, focusing on lac $Z$ reporter gene expression in the $A p o E^{-/-}$mice. Xgal and immunohistochemistry staining was performed of adventitial and lesion areas with antibodies specific for endothelial cells (VWF), SMC (ACTA2), neuronal cells (TUBB3), adipocytes (PLIN1), fibroblasts (THY1), stem cells (nestin), macrophages (LAMP2), hematopoietic cells (PTPRC), and dendritic cells (Itgax) (S7 Fig). These studies failed to identify co-

localization with known cellular lineage markers, and when combined with published negative data for pericyte markers argue that $T c f 21$ is not expressed by a common terminally differentiated vascular cell type [17].

Further reporter gene and immunohistochemistry studies focused on the fibrous cap in lesions of mice fed HFD for 4 to 20 weeks. Studies with SMC marker Tagln again revealed that the majority of Tcf21 expressing cells in the adventitia, media and plaque of diseased vessels did not express SMC markers, which were easily identified in association with medial and fibrous cap SMC (Fig 4). Although individual Tcf21 expressing cells and those in subcapsular structures did not express SMC markers, there were Tcf21 expressing cells identified immediately below or within the fibrous cap that appeared to also express Tagln. In addition to SMC lineage markers, we investigated expression of growth factor receptor Tgfbr2, which is involved in SMC development and structural vascular wall disease $[27,28]$ and was identified as one of the differentially expressed genes in the TCF21 knockdown RNA-Seq studies, as well as Pdgfrb which has an important role in coronary SMC development and atherosclerotic disease [5,29]. Fibrous cap cells were shown to express growth factor receptors Tgfbr2 and Pdgfrb, and there appeared to be co-staining for Tcf21 expression and growth factor expression by cells in the vicinity of the fibrous cap. Whether the observed co-staining identifies a unique transitional cell that expresses Tcf21 and SMC marker genes, or is due simply to sustained enzymatic activity of the $\beta$-galactosidase protein after $T c f 21$ expression is downregulated was not clear from these studies. However, these data suggest that Tcf21 expressing cells can adopt an SMC phenotype and contribute to the fibrous cap.

\section{Tcf21 expressing cells give rise to smooth muscle cells in the fibrous cap of $A p o E^{-/-}$mice}

In the next series of experiments we sought to more clearly identify the possible origins and cellular fate of Tcf21 expressing cells found in the vascular disease lesions. For these studies we employed a lineage tracing approach with an inducible Cre recombinase that provided permanent fluorophore marking of cells that expressed Tcf21 at the time of activation of recombinase expression. We used a mouse line expressing a Cre recombinase protein fused to two mutant estrogen-receptor ligand-binding domains (MerCreMer) [30], under the control of the endogenous $T c f 21$ locus ( $\left.T c f 21^{i C r e /+}\right)$. This line has been well characterized and used extensively to investigate the cellular fate of $T c f 21$ expressing cells in the developing embryo [17,31]. For reporter alleles, we employed both a tandem dimer tomato (tdT) fluorescent reporter line (B6.

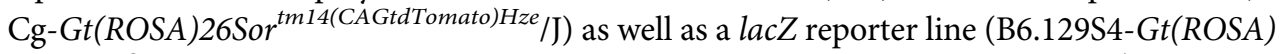
$\left.26 \mathrm{Sor}^{\mathrm{tm} I S o r} / \mathrm{J}\right)$ that was tracked with histochemical $\beta$-galactosidase activity. ApoE ${ }^{-/-}$mice carrying the Cre and reporter alleles were administered tamoxifen before HFD and sacrificed after 16 weeks of the diet, or administered tamoxifen at 6-8 weeks and harvested after 8,12 or 16 weeks of HFD before sacrifice.

Lineage tracing studies in $A p o E^{-/-}$mice were primarily conducted in animals on HFD for 12 weeks, with tamoxifen being administered from 6 to 8 weeks of the diet. At baseline, Tcf21 expression using this lineage tracing system was consistent with that seen for the lac $Z$ knockin reporter mouse line, being restricted to the adventitial cells associated with coronary arteries, 
cardiac fibroblasts, and some aortic root cells (Fig 5A). Aortic root tissues from animals treated with HFD revealed tdT-expressing cells in the lesions. For early lesions without a defined fibrous cap, Tcf21 lineage traced cells were distributed through the lesion and did not express the SMC marker Tagln (Fig 5B). In those lesions with a mature fibrous cap, Tcf21 lineage traced cells were noted to contribute to this cap and many of these cells shown to express Tagln (Fig 5C and 5D). These data are consistent with the results described above for Tcf21 reporter gene and SMC marker immunohistochemistry studies, and suggest that Tcf21 expressing cells may give rise to SMC in the fibrous cap.

By activating recombinase activity before HFD feeding and lesion development it was possible to investigate the cellular origin of the Tcf21 expressing cells identified in the vascular lesions of the $A p o E^{-/-}$model. For mice that were administered tamoxifen before HFD, and then maintained on the diet for 12 weeks, lineage traced cells were commonly identified in lesions (Fig 5E). These data are thus consistent with their derivation from Tcf21 expressing cells in the vascular wall adventitia or media, and suggest that $T c f 21$ lineage traced cells were able to migrate into the forming lesions and contribute to lesion formation.

Finally, to verify the results obtained with the fluorescent tdT reporter, we conducted identical experiments with the Cre-activated lacZ reporter mouse. These studies also found that Tcf21 lineage traced cells in the neointima did not express the SMC marker Tagln, whereas lineage traced cells contributing to the fibrous cap cells were found to express this SMC marker (S8 Fig).

\section{Lineage tracing in $\mathrm{Ldlr}^{-/-}$mice also shows recruitment of Tcf21 expressing cells that contribute to the fibrous cap}

To generalize the findings obtained in the $\mathrm{ApoE}^{-/-}$model, we conducted lineage tracing studies in $\mathrm{Ldlr}^{-/}$mice. Findings from these experiments also showed that activation of the reporter gene at 3 to 5 weeks of age, prior to HFD feeding for 16 to 20 weeks, marked cells that migrated into the atherosclerotic lesion (Fig 6A). As in the $\mathrm{ApoE}^{-/-}$model, some of the Tcf21 traced cells activated expression of SMC markers Tagln and Acta2 and localized to the fibrous cap (Fig $6 \mathrm{~B})$. In addition, co-labeling with an antibody specific for the matrix protein periostin showed some general co-localization of $T c f 21$ traced cells and periostin in the adventitia while there was extensive colocalization in the fibrous cap and co-staining in a subcap region located abluminal to the fibrous cap (Fig 6C). Also, Tcf21 traced cells in the adventitia and fibrous cap showed extensive colocalization for Pdgfra expression (Fig 6D).

\section{The rate of cell division in vascular lesions is related to Tcf21 expression}

To investigate whether Tcf21 plays a role in regulating vascular cell proliferation in the disease setting in vivo, we used two different approaches, one employing early stage disease in the $L d l r^{-/-}$model, and the other a later stage $A p o E^{-/-}$murine model. In experiments with $L d l r^{-/-}$ mice we investigated whether there was a time-dependent increase in the relative number of $T c f 21$ expressing cells that were undergoing cell division. In these studies, $T c f 21^{i C r e /+}, R O S A^{t d T /}$ ${ }^{+}, \mathrm{Ldlr}^{-/-}$mice were treated with tamoxifen at 1 week before isolation, and EdU was injected 24 hours prior to tissue harvest at relatively early disease time points for this model, at $0,9,12$, and 15 weeks of feeding with HFD. EdU positive cells were found in the adventitia, media and intima of the aortic wall and quantified in sections of the aorta through the region of the sinus. The percentage of proliferating $T c f 21$ expressing cells was identified by both tdT and EdU fluorescence (Fig 7A). Comparison of the number of Tcf21+EdU+ cells versus all $T c f 21+$ cells at different time points showed a significantly greater percentage of proliferating cells at 12 weeks of diet compared to time zero $(1.94 \pm 0.70$ vs. $6.42 \pm 1.10, P<0.05)$ (Fig $7 \mathrm{~B})$. There was also an 
A

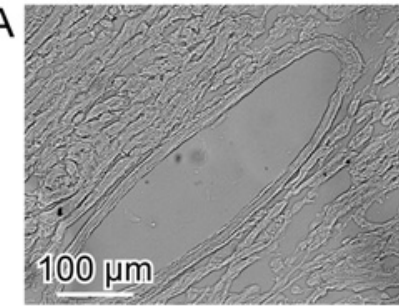

B
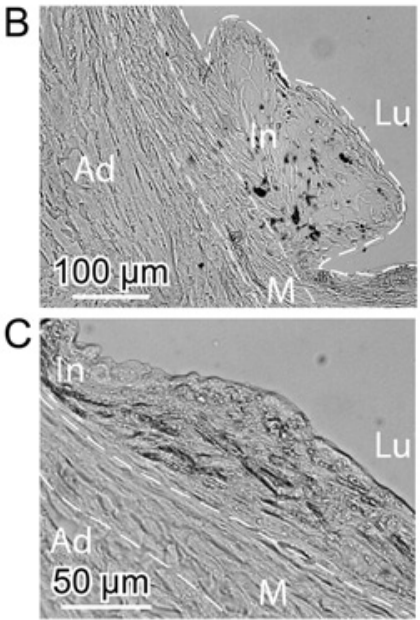

D

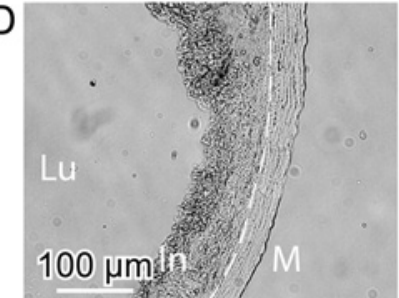

$\mathrm{E}$

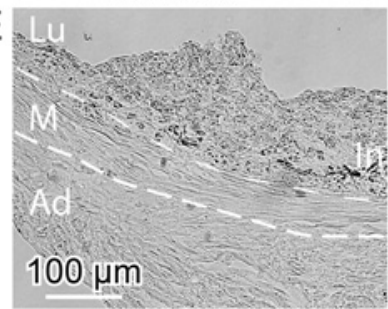

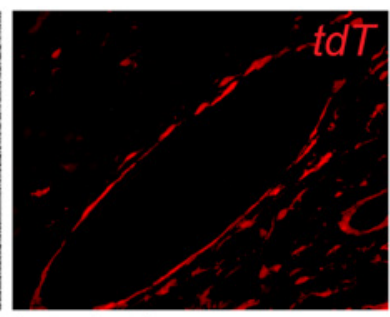
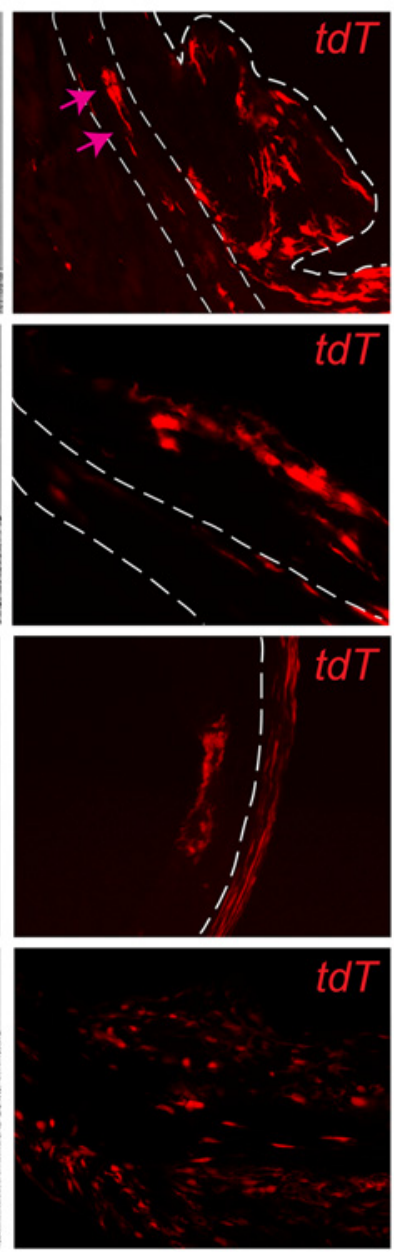
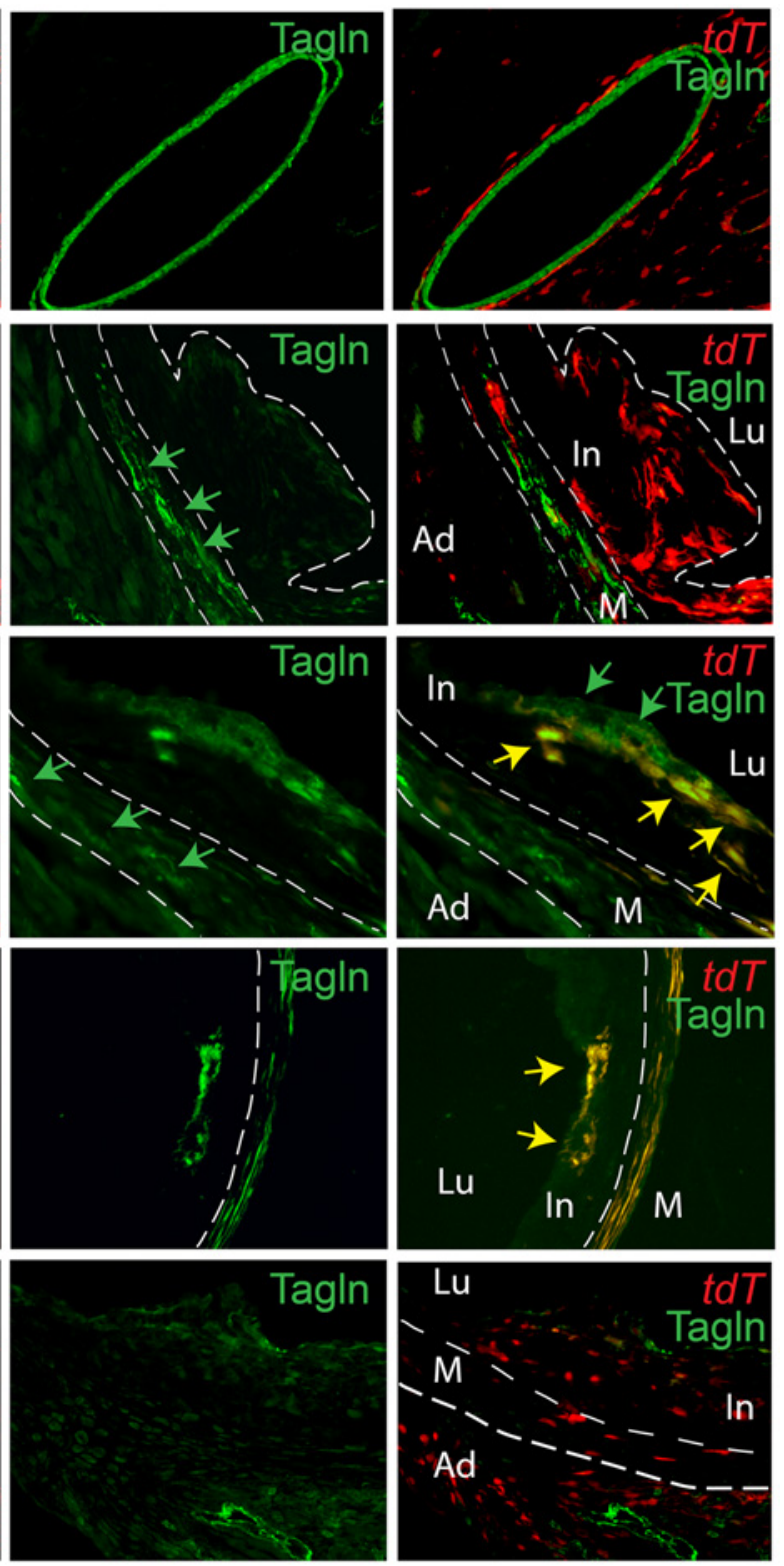

Fig 5. Tcf21 expressing cells in $\mathrm{ApoE}^{-/-}$lesions give rise to smooth muscle cells in the fibrous cap. Tcf2 $1^{i \mathrm{Cre} /+}, \mathrm{ApoE}^{-/-}$mice were administered tamoxifen to activate expression of an inducible MerCreMer construct knocked into the Tcf21 locus [31]. Cre mediated recombination of a tomato (tdT) reporter at the constitutively expressed ROSA26 locus allowed lineage tracing of Tcf21 expressing cells. Animals received tamoxifen at 6-8 weeks of HFD and tissues were harvested at 12 weeks of diet (A-D), or received tamoxifen 4-6 weeks of age prior to HFD and tissues harvested at 16 weeks (E). A) Intramyocardial coronary artery showing Tcf21 expression (tdT) in adventitial cells surrounding the Tagln positive medial SMC. B) In an early lesion without a defined fibrous cap, tdT positive cells were seen throughout the lesion and in the media (pink arrow), with Tagln staining (green arrows) being restricted to cells in the media. C) In the Tagln and tdT merged imaging (right panel), lesion Tagln positive cells are identified in the fibrous cap (green arrows) and some of these cells co-stain for tdT fluorescence (yellow arrows), indicating that they previously expressed Tcf21. Tagln expressing cells in the media (green arrows, Tagln only imaging) do not colocalize with tdT staining in this location. D) Tagln positive cells in the fibrous cap were also positive for tdT fluorescence, suggesting that the identified SMC had expressed Tcf21. Also, co-staining of tdT and Tagln is noted in the media (yellow color). E) Animals received tamoxifen at 4 weeks of age prior to HFD and tissues were harvested at 12 weeks of diet. In an early lesion without a defined fibrous cap, tdT positive cells are seen throughout the adventitia, in the media and in the plaque. In, intima; M, media; Ad, adventitia; Lu, lumen.

doi:10.1371/journal.pgen.1005155.g005 

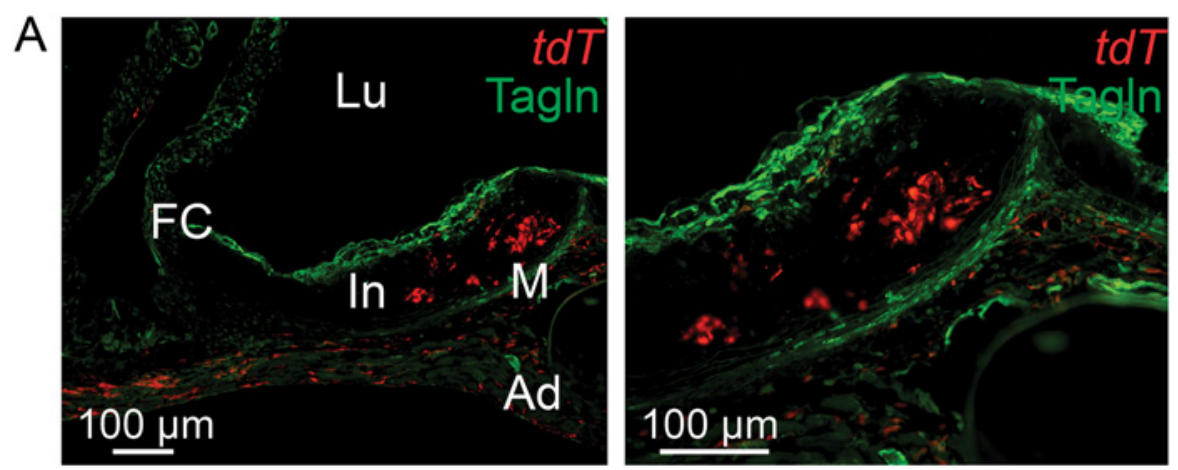

$\mathrm{B}$
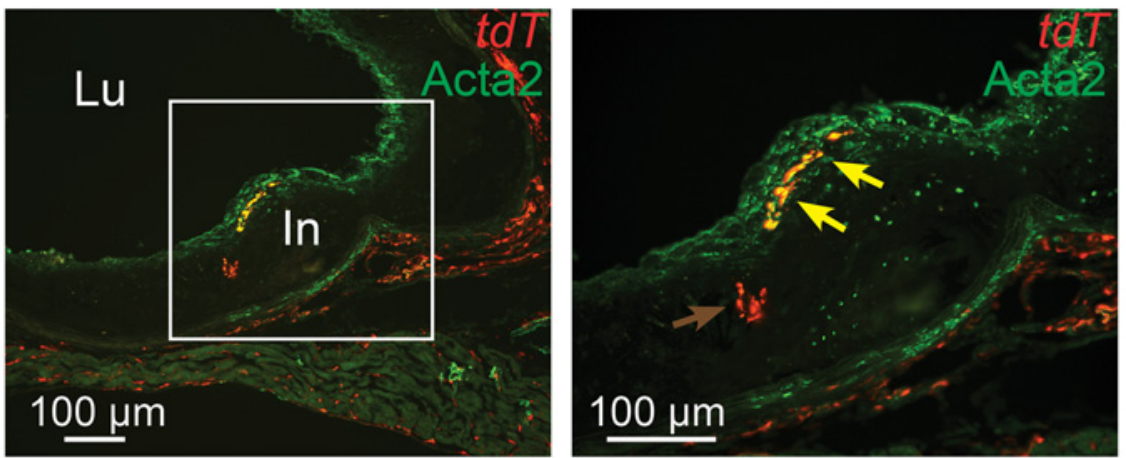

C
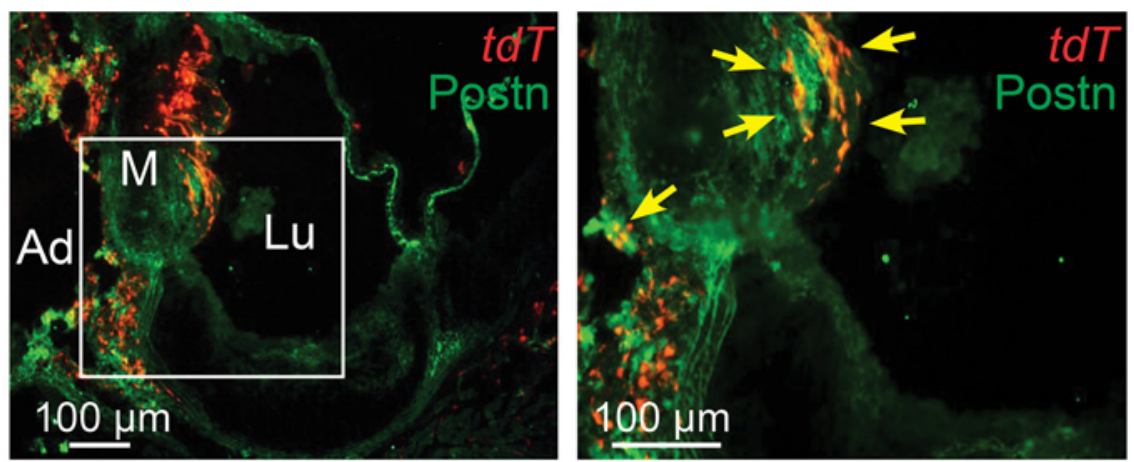

$\mathrm{D}$

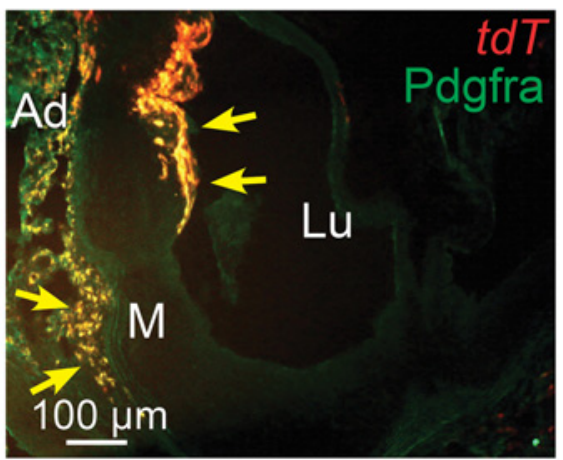

Fig 6. Tcf21 lineage traced cells in LdIr ${ }^{-/-}$lesions give rise to smooth muscle cells in the fibrous cap. $T c f 21^{i C r e /+}, L_{d l l} r^{-1-}$ mice were administered tamoxifen to activate expression of an inducible MerCreMer construct knocked into the Tcf21 locus to produce recombination and thus expression of a floxed STOP tandem dimer tomato $(\mathrm{tdT})$ reporter gene. Animals received tamoxifen chow at 3-5 weeks of age and were subsequently fed HFD for 16-20 weeks before sacrifice. A) tdT positive cells are seen throughout the lesion and in the adventitia with Tagln staining cells identified in the media and fibrous cap. B) A number of tdT lineage traced cells in the fibrous cap express the SMC marker Acta2 as shown by colocalization of fluorescent markers (yellow arrows). tdT positive cells within the lesion did not express Tagln, as shown by red color (red arrow). C) Some cells in the adventitia stain for periostin expression and colocalize with tdT staining in this location (yellow arrows). Staining for periostin and colocalization with Tcf21 lineage cells was 
also observed in the fibrous cap and subcap (yellow arrows). D) Extensive colocalization was noted for Pdgfra antibody staining and tdT fluorescence in the adventitia and fibrous cap. In, intima; M, media; Ad, adventitia; FC, fibrous cap, Lu, lumen.

doi:10.1371/journal.pgen.1005155.g006

increase in the mean percentage of EdU+Tcf21+ cells to EdU+ cells between the 9 and 12 week time points, although this difference did not reach statistical significance $(P=0.053)$. Studies also investigated whether there was a difference in the relative rate of cell division in lesions of $T c f 21^{\text {lacZ/+}}, A p o E^{-/-}$mice compared to ApoE $E^{-/-}$animals, after 20 weeks of HFD. All mice were injected with EdU 24 hours prior to tissue harvest and the number of EdU fluorescent labeled cells was compared to the total number of cells as identified by DAPI fluorescence for each genotype. This analysis was restricted to the plaque area where the majority of EdU+ cells were located in these more mature lesions (Fig 7C and 7D). The Tcf2 $21^{\text {lacZ/+ }}, A p o E^{-/-}$mice showed a significantly lower number of EdU+ cells compared to $A p o E^{-/-}$animals ( $2.25 \pm 0.18$ vs. 3.27 $\pm 0.25, \mathrm{P}<0.05$ ) (Fig $7 \mathrm{E}$ ). Taken together, these data suggest that $T c f 21$ expressing cells show a time-dependent increase in proliferation with disease progression, possibly greater than other cells not expressing Tcf21, and that this increase may depend on Tcf21 expression.

A

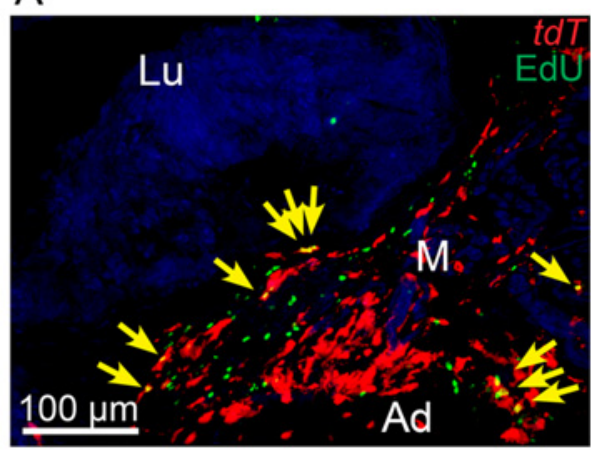

B

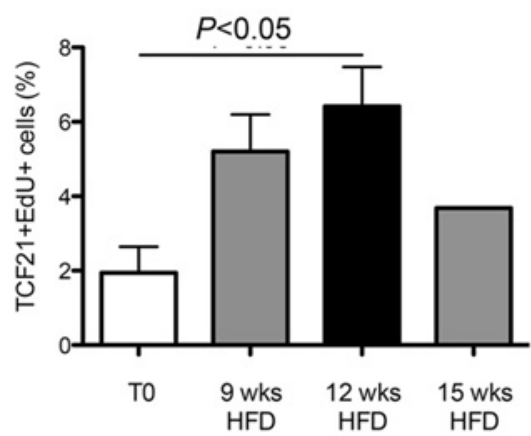

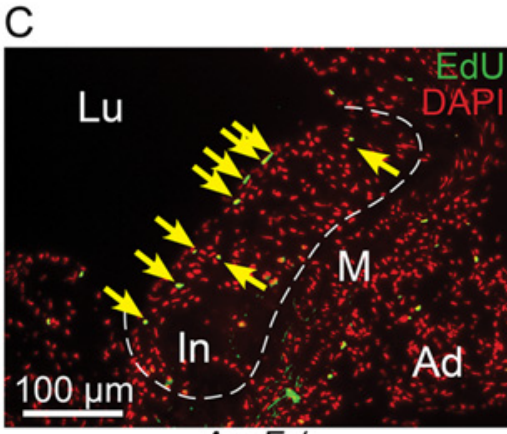

ApoE-/-
D

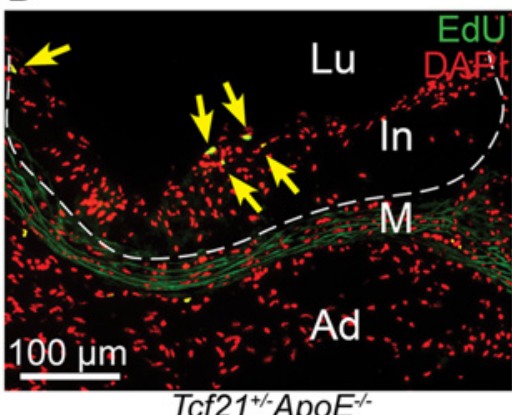

$\mathrm{E}$

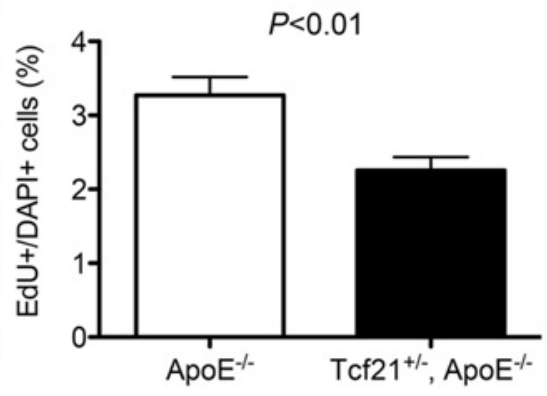

Fig 7. Rate of cell division in vascular lesions is related to Tcf21 expression. A) EdU staining was performed in Tcf $21^{i C r e /+}, R O S A^{t d T /+}, L d l r^{-/-}$mice treated with tamoxifen and EdU and placed on HFD for 9, 12, or 15 weeks prior to tissue collection. Co-localization of Tcf21 tdT fluorescence (red) and EdU fluorescent staining (green) allowed identification of the sdividing cells that were expressing Tcf21 (yellow arrows, image from 12 week timepoint). B) The ratio of $T c f 21+E d U$ + cells compared to total Tcf21+cells was significantly increased at 12 weeks of diet, consistent with a proliferative response in Tcf21 + cells. Comparison of percentage differences between 0 and 9 weeks, and from 9 to 12 weeks approached significance, $P=0.06$; there was not a statistical difference between 12 and 15 weeks, $P=0.11$. C, D) Rates of cell division in vascular lesions were compared between $T c f 21^{l a c Z /+}, A p o E^{\prime-}$ animals and $A p o E^{-/}$animals on HFD for 20 wks by quantifying the relative number of dividing cells identified by EdU fluorescence compared to the total number of cells identified by DAPI fluorescence. EdU fluorescence (green) was merged with red pseudocolored DAPI fluorescence, yellow arrows indicate yellow nuclei that

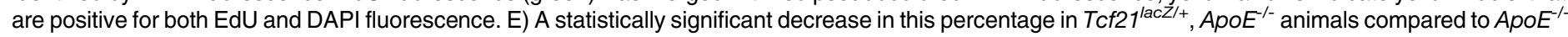
animals suggested a correlation between Tcf21 expression and rate of cell division in the vascular lesions. In, intima; M, media; Ad, adventitia; FC, fibrous cap, Lu, lumen. 

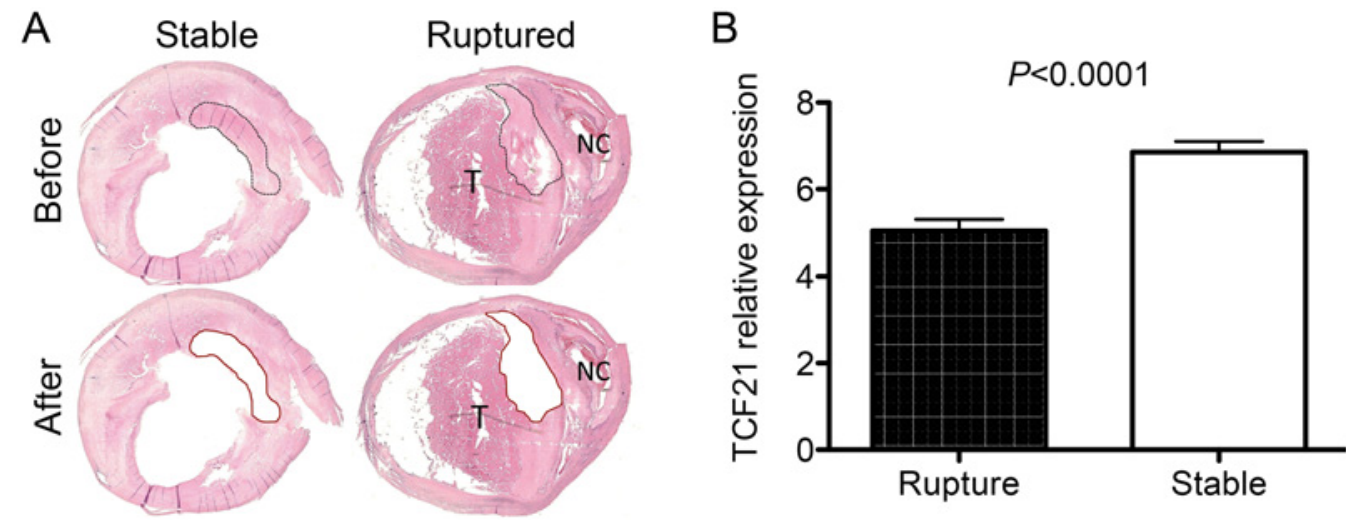

Fig 8. Differential TCF21 gene expression in fibrous cap of stable vs. ruptured atherosclerotic plaque. A) Microdissection laser capture was employed to harvest tissue from endarterectomy samples from vessels with stable plaque or evidence of plaque rupture. An example of a stable plaque and a ruptured plaque with necrotic core (NC) and related luminal thrombus $(\mathrm{T})$ are shown. These representative sections show the types of plaque regions that were harvested for RNA isolation. B) qPCR quantitation of TCF21 mRNA levels in harvested tissue. TCF21 expression was significantly lower in fibrous cap tissue from the ruptured plaque.

doi:10.1371/journal.pgen.1005155.g008

\section{TCF21 is differentially expressed in the fibrous plaque of ruptured human atherosclerotic lesions}

To begin to investigate the possible association of TCF21 expression and function in the fibrous cap of human atherosclerotic lesions, we have investigated the TCF21 expression pattern in fibrous plaque of carotid atherosclerotic lesions collected from patients at time of endarterectomy surgery. Micro-dissection laser capture was used to obtain tissue from the fibrous cap of 10 vessels that showed stable plaque morphology and 10 vessels that had undergone plaque rupture (Fig 8). RNA was isolated from these tissues and employed to assess TCF21 mRNA levels by qPCR. This analysis showed significantly decreased levels of TCF21 expression in the fibrous cap tissue harvested from the ruptured plaque (5.0 \pm 0.3 vs $6.9 \pm 0.3$, ruptured vs stable plaque, $P<0.0001)$.

\section{Discussion}

SMC are critical contributors to atherosclerosis pathophysiology, and relevant to the findings reported here is the dramatic alteration in gene expression and basic cellular functions that occur with phenotypic modulation of this cell type in the disease setting $[5,32,33]$. In the classic response to injury hypothesis, fully differentiated medial SMC express contractile marker genes and have limited proliferative capacity, while under disease conditions they downregulate contractile gene expression, proliferate, and migrate into the neointimal space. Data presented here suggest that upregulation of TCF21 may be directly responsible for at least some aspects of the phenotypic modulation that is believed to characterize the medial SMC response to vascular injury. We have shown that TCF21 binds the ACTA2 locus, transrepresses ACTA2 reporter genes in cultured SMC, and that knockdown of TCF21 is associated with increased ACTA2 expression. Although we have not studied other SMC markers in such detail, both TAGLN and MYH11 are upregulated in siTCF21 treated cells. While additional studies are required to characterize a possible dedifferentiation function for TCF21, also consistent with such a hypothesis are in vitro studies in cultured HCASMC presented here which show that TCF21 promotes proliferation and migration. A differentiation inhibiting function has previously been proposed for TCF21 in skeletal muscle cells [34]. 
Tcf21 is expressed in epicardial progenitor cells that give rise to coronary SMC, and its expression in the adult might be characteristic of a precursor cell that is resident in the vascular wall and is capable of giving rise to SMC in the setting of vascular injury. A number of previous studies have identified such precursor cells in distinct compartments of the vessel wall, all with some capacity to give rise to differentiated SMC. It is generally accepted that SMC accumulation in the fibrous cap is monoclonal or oligoclonal, and this is consistent with the existence of a resident arterial subpopulation of SMC precursor cells that contribute to healing in the response to disease stresses [35]. In bovine and canine vessels, subsets of medial SMC have prominent proliferation with reduced SMC marker expression, and this population has been estimated to be $6-15 \%$ of cells in the murine aorta [36-38]. These observations are consistent with data presented here showing that Tcf21 expressing cells with low SMC marker expression are distributed in the proximal aorta in mouse. Cells with SMC precursor function have also been identified in the adventitial layer [35]. In mouse, $\mathrm{Scal}^{+}{ }^{+}$cells with SMC differentiation potential have been identified in the aortic adventitia [39], and in humans, a population of cells with SMC potential has been identified in the space between the media and adventitia of large and medium sized vessels [40]. While there are reported differences in the features and markers of these and other progenitor-like cells in the vascular wall, here we demonstrate an overlap of expression pattern and other features in Tcf21 expressing cells. It seems likely that TCF21 may be expressed in one or more of these cell populations and may contribute to an undifferentiated SMC precursor phenotype.

The studies described herein provide important new information regarding the biology of the fibrous cap. While the risk of plaque rupture appears to be inversely correlated with the number of SMC in the fibrous cap, there is limited understanding of their in vivo origin, and the molecular pathways that regulate their expansion and terminal phenotype determination [5,41]. With these studies we have shown $T c f 21$ to be a marker for precursor cells that give rise to at least a portion of fibrous cap cells, and genomic studies in conjunction with in situ protein marker studies identified a number of signaling molecules that relate to the phenotype of this important cell type. While Pdgfra and Pdgfrb have been previously identified on fibrous cap cells $[42,43]$, we show here that they are not expressed by cells that are migrating toward the cap and expressing Tcf21 but initiate expression in conjunction with upregulation of SMC marker genes when they are juxtaposed to the endothelium. Tgfbr2 has not previously been identified on fibrous cap cells, and its biology in this context not studied, but it has recently been shown to support the differentiated SMC phenotype and homeostasis of this cellular lineage $[27,28]$. TGFBR 2 mRNA levels were found to be regulated by TCF21 in the siRNA knockdown RNA-Seq studies. Another protein expressed by Tcf21 lineage-traced cells in the fibrous cap, periostin, is known to promote SMC differentiation and migration [44-46], and interestingly has recently been associated with the atherosclerotic phenotype in young humans [47]. Additional follow-up of genomic studies are expected to provide insights into other genes and pathways that are present in TCF21 marked fibrous cap precursor cells.

Lineage tracing provides a unique and informative approach to address questions related to cellular origin, movement and differentiation of lesion cells in the vascular wall, however a number of questions remain unanswered. Given the capacity of Tcf21 to promote autonomous cellular proliferation and migration, and the appearance of lineage traced cells in lesions of animals where recombination preceded HFD feeding, it seems likely that lineage traced cells can migrate from the adventitia or media to contribute to lesion formation. Although there is some evidence that bone marrow-derived SMC-like cells may contribute to neointima formation, such cells appear to represent a very small fraction of the total neointimal cells $[48,49]$. Given the variability in disease progression in different $A p o E^{-/}$and $L d l r^{-/}$animals, and the possibility that $T c f 21$ is upregulated in adventitial or medial cells throughout disease progression, lineage 
tracing does not allow assessment of how much of the fibrous cap derives from Tcf21 expressing cells. Results from the $T c f 21^{l a c Z /+}, A p o E^{-/-}$studies suggest that $T c f 21$ expressing cells constitute a significant portion of the lesion SMC but this cannot be readily evaluated with current methods. Also, evidence that some fibrous cap SMC derive from lineage traced cells does not eliminate the possibility that a portion of the fibrous cap cells continue to express $T c f 21$ and maintain a fibroblast or myofibroblast phenotype, and that the fibrous cap is actually a combination of such cells along with those that have downregulated Tcf21 and adopted a true SMC phenotype. This possibility is consistent with sustained Tcf21 expression in some of the fibrous cap cells at the 20-week disease timepoint (Fig 3).

While the work reported here has provided important initial details of how this gene is expressed during atherosclerotic vascular disease and the types of processes that it might regulate to affect disease pathophysiology, the causal mechanisms of disease risk remain unclear. The direction of effect has not been fully established by current available human genetic and genomic data, with both directions being suggested by different approaches and possible underlying gene regulatory mechanisms characterized for each $[8,50,51]$. Based on the animal model findings reported here, greater TCF21 expression could inhibit disease risk by promoting the expansion of SMC lineage and contributing to plaque stabilization by supporting fibrous cap development. Alternatively, greater TCF21 expression in SMC precursor cells at the fibrous cap might inhibit their differentiation to the SMC lineage, thus destabilizing the plaque. Similar alternative hypotheses could be generated for the risk allele producing decreased TCF21 expression. Nonetheless, further in vivo gain- and loss-of-function studies are required to determine how changes in $T c f 21$ gene expression affects the size and architecture of the fibrous cap and how such changes correlate to specific human disease traits linked to plaque vulnerability and rupture. These questions could be addressed using the various plaque rupture models established in mice $[52,53]$. Finally, it will be important to investigate the phenotype of the TCF21 expressing cells at baseline, and how the phenotype changes as these cells migrate into the lesion. Such studies could be performed with captured cells using modern genomic methods, and would provide extremely valuable insights into the cellular phenotype as these cells respond to the vascular signals of the disease process.

\section{Methods}

\section{Ethics statement}

All procedures described in this study were approved by the Institutional Animal Care and Use Committees of Stanford University and University of Hawaii and conformed to NIH guidelines for care and use of laboratory animals.

\section{Cell culture}

Human primary Coronary Artery Smooth Muscle Cells (HCASMC, \#CC-2583) were obtained from Lonza (Allendale, NJ, USA) at passage 4 and cultured in Smooth Muscle Growth Medium-2 including hEGF, insulin, hFGF-B and FBS, but without antibiotics (Lonza, \#CC-3182). Human embryonic kidney cells (HEK, ATCC \#CRL-11268) and rat aortic smooth muscle cells (A7R5, ATCC \#CRL-1444) were cultured in DMEM High Glucose (Life Technologies \#311995-065) with 10\% FBS (Life Technologies \#26140-79). Human cardiac fibroblast (HCF), bone-marrow derived mesenchymal stem cells (bmMSC), embryonic stem cell derived mesenchymal stem cells (eMSC), induced pluripotent stem cell-derived mesenchymal stem cells (iMSC), human aortic adventitial fibroblasts (HAoAF), human aortic endothelial cells (HAoEC), human coronary artery endothelial cells (HCAEC), and human aortic smooth 
muscle cells (HAoSMC) were obtained from commercial suppliers, maintained in the recommended media, and evaluated with at least 3 technical replicates.

For RNA-Seq studies and individual gene quantitative PCR, HCASMC were transfected with 300 nM TCF21 Trilencer-27 Human siRNA (OriGene \#SR304753C) or Trilencer-27 Universal Scrambled Negative Control siRNA (OriGene \#SR30004) at $80 \%$ confluence using the Amaxa Basic Nucleofector Kit for Primary Mammalian Smooth Muscle Cells (Lonza \#VPI1004) at a density of $1 \times 10^{6}$ cells per $100 \mu \mathrm{L}$ sample using Nucleofector Program U-025. Cells were changed to medium with supplements at 18 hours post-transfection and cultured for an additional 48 hours.

\section{Lentivirus construction and in vitro cell culture assays}

For TCF21 overexpression, a cDNA clone (OriGene \#SC125048) representing the protein coding sequence of human TCF21 was cloned into pWPI vector (Addgene \#12254) which also contains the GFP reporter gene. For shRNA knockdown the following sequences were cloned into pLVTHM (Addgene \#12247):

shTCF21 construct 1. 1:GaattcgaacgctgacgtcatcaacccgctccaaggaatcgcgggcccagtgtcactaggcgggaacacccagcgcgcgtgcgccctggcaggaagatggctgtgagggacaggggagtggcgccctgcaatatttgcatgtcgctatgtgttctgggaaatcaccataaacgtgaaatgtctttggatttgggaatcttataagttctgtatgagaccacagatctCCCTGGATTCGAACAAGGAATTTGTGACTTTTCAAGAGAAAGTCACAAATTCCTTGTTCGAATCCATTTTTGGAAaagcttATCGAT

shTCF21 construct 2. GaattcgaacgctgacgtcatcaacccgctccaaggaatcgcgggcccagtgtcactaggcgggaacacccagcgcgcgtgcgccctggcaggaagatggctgtgagggacaggggagtggcgccctgcaatatttgcatgtcgctatgtgttctgggaaatcaccataaacgtgaaatgtctttggatttgggaatcttataagttctgtatgagaccacagatctCCCTGGAGATGTTGGAATGTGACGGGTTGATTCAAGAGATCAACCCGTCACATTCCAACATCTCCATTTTTGGAAaagcttATCGAT

Lentiviruses were produced by polyethylenimine (Polysciences cat \#23966-2)-mediated transfection of 293T cells (American Type Culture Collection \#CRL-11268). Per $225 \mathrm{~cm}^{2}$ flask, $3.6 \times 10^{7}$ cells were plated 24 hours before transfection in DMEM (Invitrogen cat \#11995), 10\% fetal bovine serum (Hyclone cat \#SH30070.03). For transfection, $30 \mathrm{mg}$ of lentivirus vector, $30 \mathrm{mg}$ of a HIV-1 gag-pol-rev-tat helper plasmid (pCMV DR8.9, Addgene \#8455), and $15 \mathrm{mg}$ of a plasmid encoding the VSV-G envelope protein (pMD2.G, Addgene \#12259) were used per T-225 flask of cells transfected. The plasmids ( $75 \mathrm{mg}$ total) were added to OptiMEM (Invitrogen cat \# 31985) such that the total volume was $700 \mathrm{ml}$ and then $300 \mathrm{ml}$ of $1 \mathrm{mg} / \mathrm{ml}$ polyethylenimine ( $25 \mathrm{kDa}$ linear, $\mathrm{pH} 4.5$ in $1 \mathrm{x}$ PBS) was added. The PEI/DNA mixture was mixed, incubated $10 \mathrm{~min}$ at $20^{\circ} \mathrm{C}$, and then added to the cells. The cells were incubated at $37^{\circ} \mathrm{C}$ in a $5 \%$ $\mathrm{CO}_{2}$ atmosphere. Then 24 and 48 hours after transfection the media was harvested and replaced with fresh media. After harvesting the media, dead cells were removed by centrifugation at 3,000 $\mathrm{x}$ g for $15 \mathrm{~min}$. The virus was filtered through a $0.45 \mu \mathrm{m}$ Steri-flip filter (Millipore cat \#SE1M003M00) and then aliquoted and stored at $-80^{\circ} \mathrm{C}$ until use.

HCASMC were transduced with lentiviral particles at a MOI of 22.5 at passage 7 to 9 . The transduction efficiency was assessed by quantifying the percentage of GFP-positive cells by flow cytometry on a BD FACSCalibur. Transduction efficiencies were between 50 and 95 percent.

Cell migration (gap closure) assay. The gap closure assay (Cell Biolabs \#CBA-126) was conducted according to the manufacturer's protocol. Briefly, 10,000 lentivirus transduced cells were seeded per well and let attach overnight. After gel removal 3 wells per condition were directly stained with crystal violet and imaged using a Zeiss Axioplan 2 instrument using the NIS-Elements F v4.0 software while the remaining 9 wells per condition were incubated for $12 \mathrm{~h}$ before crystal violet staining. The covered area per well was quantified using ImageJ v1.47. 
In vitro proliferation assay (cell counting). Lentivirally transduced HCASMC were plated into 6 well plates at low density (10,000 cells per well, 3 wells per condition) and harvested at given time points before reaching confluence. Viable cell numbers were then counted manually and percentage of GFP positive cells measured by flow cytometry.

In vitro proliferation assay (EdU labeling). Lentivirus transduced cells were plated to a 12 well plate at sub-confluence density and allowed to recover overnight. The cells were then serum starved for 24 hours. Following starvation, the cells were exposed to serum for $24 \mathrm{hrs,}$ with treatment during the last $3 \mathrm{hrs}$ of this period with EdU from the Click-iT EdU Alexa Fluor 488 Imaging Kit (Life technologies, Carlsbad, CA; Cat\# C10377) at a concentration of $20 \mathrm{uM}$. The cells were incubated with EdU for 3 hours, then fixed and permeabilized using 4\% PFA and $0.5 \%$ Triton-X in PBS, respectively. This was followed by incubation with Click-iT reaction cocktail, including CuSO4 and Alexa Fluor Azide, and then with nuclear staining with DAPI solution. Using a Leica inverted microscope, the number of total nuclei, and the number of co-stained nuclei were counted using the Image (NIH) software on ten consecutive 10x fields for each condition.

In vitro apoptosis assay. Cells were transfected with siControl or siTCF21 reagents exactly as described above for the RNA-Seq studies, at a density of $1 \mathrm{E} 6$ cells per $100 \mu \mathrm{L}$ sample using Nucleofector Program U-025. Cells were changed to medium without supplements at 18 hours post-transfection and cultured for an additional 48 hours. Caspase activity in these cells was detected using the Caspase-Glo 3/7 Assay (Promega \#G8093).

\section{RNA isolation and quantitative real-time RT-PCR}

For qPCR RNA was isolated using QIAzol (Qiagen \#79306) and the Trizol RNA isolation protocol. DNA was removed with the DNA-free kit from Ambion (Life Technologies \#AM1906) followed by first strand cDNA synthesis and subsequent RNA digestion according to the SuperScript III protocol (Life Technologies \#18080-051). Relative gene expression was measured using the ViiA 7 Real-Time PCR System from Applied Biosystems (Life Technologies). cDNA was amplified as follows: $95^{\circ} \mathrm{C} 15 \mathrm{~min}, 40 \mathrm{x}\left(95^{\circ} \mathrm{C} 1 \mathrm{~s}, 60^{\circ} 20 \mathrm{~s}\right)$. Locus enrichment in chromatin immunoprecipitation samples was measured using the ABgene SYBR mastermix (AB-1166) and the primers ACTA2chipfor 5'-AGGGAGATGCAAACCAGATATCC-3', ACTA2chiprev 5'-GCAGGTGACCTGCTGAATTTTTC-3', PROCRchipfor 5'-GACTCCTGCTTACCTCCTCATA-3' and PROCRchiprev 5'-GGGTGAAGAAGGTACAAAAGAA-3'. Genomic DNA cycling conditions were $95^{\circ} \mathrm{C} 15 \mathrm{~min}, 40 \mathrm{x}\left(95^{\circ} \mathrm{C} 15 \mathrm{~s}, 60^{\circ} \mathrm{C} 1 \mathrm{~min}\right)$. Absolute quantification of expression levels was performed using a standard dilution series and normalization to $18 \mathrm{~S}$ rRNA. Relative expression levels were then calculated relative to TCF21 expression levels in HEK cells (S1A Fig) or in SiCTRL treated HCASMC (Fig 2E) by division.

\section{RNA sequencing}

Total RNA from either siTCF21 or siCTRL treated samples was depleted for ribosomal RNA with the Ribo-Zero magnetic kit from Epicentre (Illumina \#MRZH116), libraries generated with the Epicentre ScriptSeq v2 RNA-Seq library preparation kit (Illumina \#SSV21106) and thereafter sequenced as 100bp paired-end reads on an Illumina HiSeq 2000 instrument. The resulting data has been deposited at GEO under accession number GSE44461.

Reads resulting from RNA-Sequencing of siCTRL and siTCF21 treated HCASMC were mapped using software tools TopHat+Bowtie2. Differential expression level between samples was analyzed using the software tools DESeq and edgeR at an FDR $\leq 0.05$, with intersection of the 466 and 430 respective identified genes providing a group of 380 common genes. This genelist was used to interrogate the Ingenuity Knowledge Base, identifying over-representation 
of Cardiovascular Disease annotation terms, including the top functional category "Vascular disease." Gene members of this group were expanded by adding 18 genes with the "build" function in IPA to create the TCF21 Vascular Disease Network. Visualization of the network was performed using Cytoscape open source software. Node color was mapped to log fold change (green-yellow-red palette), node size to absolute expression value in wild type cells, and font size to enrichment $Q$-value. GO categories were assigned to genes using the Bingo application for Cytoscape and coloring the GO categories was performed using GOlorize application for Cytoscape.

\section{Mouse models}

A $T c f 21$ functional knockout and reporter line, $T c f 21^{\text {lacZ/+ }}$, was constructed by substituting the lac $Z$ gene into the first exon of the murine gene employing homologous recombination in embryonic stem cells, as described previously [12]. Fidelity of expression of the lacZ reporter has been described [12], and methods for using Xgal staining to track Tcf21 expression were identical to those published previously from this laboratory [54]. For studies of reporter gene expression in the $\mathrm{ApoE}^{-/-}$model, animals were weaned onto Western high fat diet (HFD, 21\% anhydrous milk fat, $19 \%$ casein and $0.15 \%$ cholesterol, Dyets no. 101511). The animals were euthanized and perfused with PBS followed by $0.4 \%$ PFA, the aortic roots excised and subjected to Xgal staining followed by post-fixation for 30 minutes in $4 \%$ PFA. After incubation in an ascending sucrose solution series and embedding in OCT, tissue was processed into $7 \mu \mathrm{m}$ thick frozen sections for immunohistochemistry.

Lineage tracing studies employed a recently described inducible Tcf21-Cre line ( $\left.T c f 21^{i \mathrm{Cre} /+}\right)$

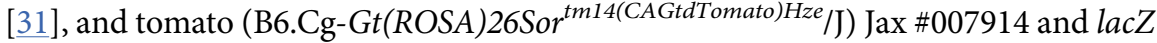
(B6.129S4-Gt(ROSA)26Sor ${ }^{\text {tmISor } / J) ~ J a x ~ \# ~} 003474$ reporter lines. The Tcf2 ${ }^{i \mathrm{Cre} /+}$ line was obtained on a mixed $\mathrm{C} 57 \mathrm{Bl} / 6 \times 129 \mathrm{SV}$ mixed background and was bred back onto the $\mathrm{C} 57 \mathrm{Bl} / 6$ background for 6 generations before being mated with the $\mathrm{ApoE}^{-/-}$line. The $T c f 21^{i \mathrm{Cre} / \mathrm{t}}$ allele was bred directly onto the $\mathrm{Ldlr}^{-/-}$line in $\mathrm{C} 57 \mathrm{Bl} / 6$ background. All $\mathrm{Ldlr}^{-/-}$mice were treated with a modified Western high fat diet (HFD, 15.8\% milk fat and 1.25\% cholesterol (diet 94059; Harlan Teklad) for the time periods indicated. Tcf $21^{i C r e /+}, R_{O S A}^{t d T /+}, A p o E^{-/} ; T c f 21^{i C r e /+}, R O S A^{t d T /+}$, $\mathrm{Ldll}^{--}$; and $\mathrm{Tcf} 2 \mathrm{I}^{\mathrm{iCre/+}}, \mathrm{ROSA}^{\mathrm{lacZ/+}}, \mathrm{ApoE}^{-/-}$and $\mathrm{Ldlr}^{-/-}$and $\mathrm{ApoE}^{-/-}$control animals were treated as described with HFD, tissues harvested and sectioned as described above, and analyzed for expression of the reporter genes as well as for other cellular markers as described below. For ApoE ${ }^{-/}$animals, Cre recombination was activated with a series of ten $1 \mathrm{mg}$ tamoxifen (Sigma Aldrich) intraperitoneal injections for a total of $10 \mathrm{mg}$ of tamoxifen. Animals were induced at 0 , 6,10 weeks and sacrificed after 20,8, and 12 weeks respectively of the HFD as described above. For $\mathrm{Ldlr}^{-/}$mice, Cre induction was accomplished by feeding of tamoxifen chow at 3-5 weeks of age (Harlan Laboratories, Inc).

\section{Immunostaining}

For immunocytochemistry, HCASMC were seeded into culture slides (BD Biosciences \#35641) in supplemented medium for 24 hours. For immunohistochemistry of human tissues, paraformaldehyde-fixed human coronary arteries were frozen, embedded and sectioned at a thickness of $7 \mu \mathrm{m}$ and tissue slides were postfixed with 4\%PFA in PBS for 10 minutes. Staining for TCF21 expression was conducted on frozen tissue sections with a polyclonal anti-TCF21 antibody (Sigma-Aldrich \#HPA013189) at a dilution of 1:100, with overnight primary incubation.

Mouse tissues were collected and processed as described above. After postfixation, nonspecific binding sites were blocked for 30 minutes with Rodent Block M (Biocare Medical \#RBM961). Primary antibody incubation was performed overnight at $4^{\circ} \mathrm{C}$ using the following 
antibodies and dilutions in Da Vinci Green antibody diluent (Biocare Medical \#PD900), antiActa2 1:300 (Abcam \#ab5694), anti-Tagln 1:300 (Abcam ab14106), anti-Pdgfrb 1:500 (Abcam \#ab32570), anti-Tgfbr2 1:100 (Abcam \#ab61213), anti-periostin 1:200 (Santa Cruz \#sc49480), anti-Pdgfra (R\&D Systems \#AF1062), anti-vWF 1:250 dilution (Abcam ab11713), anti-TUBB3 1:1000 dilution (Abcam ab78078), anti-PLIN1 1:500 dilution (Abcam ab61682), anti-THY1 1:200 dilution (Dianova DIA-100-M), anti-NES 1:2000 dilution (Abcam ab134017), antiLAMP2 1:150 dilution (Abcam ab37024), anti-PTPRC 1:300 dilution (Abcam ab), and antiITGAX 1:100 dilution (Abcam ab33483). The slides were incubated for $1 \mathrm{~h}$ at room temperature with Rabbit-on-Rodent AP polymer (Biocare Medical \#RMR625) and 10-15 minutes with the substrate Vulcan Fast Red (Biocare Medical \#FR805). Between steps slides were washed $2 \mathrm{x}$ 5 minutes with TBS (Sigma-Aldrich \#T6664). The slides were then dehydrated in an ascending alcohol dilution series and mounted in Cytoseal XYL (Thermo Scientific \#8312-4). Images were taken with a Zeiss Axioplan 2 using the NIS-Elements F v4.0 software.

EdU staining was employed to quantify in vivo rates of vascular wall cell proliferation in $T c f 21^{i C r e /+}, R_{O S A}^{t d T /+}, L d l r^{-/-}$mice. These mice were weaned onto HFD at 4 weeks of age, administered tamoxifen by a single gavage of $10 \mu \mathrm{g} / 40 \mathrm{~g}$ body weight 1 week before tissue harvest, and sacrificed and evaluated at T0, 9wks, 12 wks or $15 \mathrm{wks}$ of diet. 5-Ethynyl-2-deoxyuridine (Santa Cruz, $50 \mu \mathrm{g} / \mathrm{g}$ body weight) was injected intraperitoneal 24 hours before sacrifice. Aortic root tissues were harvested, processed and sectioned, and $T c f 21+\mathrm{EdU}+$ positive cells were quantified and expressed as the $\%$ of $T c f 21$ lineage cells. This percentage was evaluated for significant differences among the various time points evaluated. These data are based on three 40x images at each time point in each animal.

Similar experiments were performed to compare the relative rates of cell division in $T c f 21^{\text {lacZ/+ }}, A p o E^{-/-}$compared to ApoE $E^{-/-}$mice. Animals in each group were injected with $1 \mathrm{mg}$ of EdU (Life Technologies, cat\#A10044) 24 hours before sacrifice and EdU incorporation was imaged according to the manufacturer's instructions with the Click-iT EdU Alexa Fluor 488 Imaging Kit (Life Technologies) on OCT (Tissue-Tek) frozen sections. Eight sections were assessed from each group with ImageJ software (NIH) to determine the ratio of EdU positive cells to the total number of DAPI positive cells within plaque areas.

\section{Micro-dissection laser capture and TCF21 expression analysis in human atherosclerotic lesions}

Human atherosclerotic carotid artery lesions were obtained from patients undergoing endarterectomy surgery for stable (asymptomatic; $n=10$ ) or vulnerable (symptomatic; $n=10$ ) carotid stenosis, as part of the Biobank of Karolinska Endarterectomies (BiKE). Samples were collected with informed consent procedure and the study approved by the Local Ethical Committee. Patient demographics, symptom definition and sampling routines were described previously [55]. Micro-dissection laser capture of fibrous cap structures from both subgroups of patient samples was performed using a PALM Microlaser system (Zeiss, Germany) according to manufacturer's instruction. Atherosclerotic lesions, stable and ruptured (vulnerable), were paraffin-embedded, sectioned, and stained with hematoxylin \& eosin on RNAse-free glass slides. Sections were pre-treated with UV light at $254 \mathrm{~nm}$ for 30 minutes to overcome the hydrophobic nature of the membranes and to enhance adhesion of the paraffin-embedded sections. Ten consecutive slides per individual patient were micro-dissected and then pooled for gene expression analysis.

In preparation for laser pressure catapulting, sections were first de-paraffinized with Xylene ( $2 \times 2$ minutes) and decreasing $\mathrm{C}_{2} \mathrm{H}_{6} \mathrm{O}$ (Ethanol) concentrations (100\%, 96\%, 70\% each for 1 minute). Sections were then rinsed in RNAse-free water, and stained for 10 minutes with 
Mayer's hematoxylin (Sigma, USA), again rinsed for 3 minutes in RNAse-free water, and then consecutively stained for 3 minutes with Eosin (Sigma, USA). Finally, samples were dehydrated with increasing concentrations of $\mathrm{C}_{2} \mathrm{H}_{6} \mathrm{O}$, before briefly being air-dried at room temperature. After micro-dissection and catapulting, the fibrous cap sample was collected into AdhesiveCaps (Zeiss, Germany) and 350 $\mu$ of RLT buffer (Qiagen, Germany) was added, and mixed by inversion after closure. The lysate was spun down for 5 minutes at $13000 \mathrm{rpm}$ and stored at $-80^{\circ} \mathrm{C}$.

RNA extraction from catapulted, micro-dissected samples was performed using the RNeasy Micro Kit (Qiagen, Germany) following the manufacturer's protocol. RNA was quantified by Nanodrop (Agilent Technologies), and RNA quality was verified using an Agilent 2100 Bioanalyzer (Agilent Technologies). Samples required 260/280 ratios $>1.8$, and sample RNA integrity numbers $>9$ for inclusion. The TaqMan High Capacity cDNA Transcription Kit was used for cDNA synthesis, and primer assays for TCF21 and 18S (for housekeeping/endogenous control) were utilized to detect changes in mRNA expression levels. The mean TCF21 mRNA level (run in triplicate) was normalized to the mean $18 \mathrm{~S}$ mRNA level (run in triplicate) to calculate the change in expression for each condition.

\section{Statistical analysis}

Results are expressed as means \pm standard deviation (SD) with number (n) of replicates. Statistical comparisons of two groups were performed by two-tailed t-test using Prism if not stated otherwise. Welch's Correction for unequal variance was applied.

\section{Supporting Information}

S1 Fig. TCF21 is expressed by coronary vascular cells. A) Relative TCF21 expression levels in human cultured cells as measured by quantitative realtime RT-PCR: HEK, human embryonic kidney cell line; HCF, human cardiac fibroblast; bmMSC, bone-marrow derived mesenchymal stem cells; eMSC, embryonic stem cell-derived mesenchymal stem cells; iMSC, induced pluripotent stem cell-derived mesenchymal stem cells; HAoAF, human aortic adventitial fibroblasts; HAoEC, human aortic endothelial cells; HCAEC, human coronary artery endothelial cells; HAoSMC, human aortic smooth muscle cells; HCASMC, human coronary artery smooth muscle cells. Numbers for HCASMC data represent different donor samples. B) Immunostaining for TCF21 (green) and ACTA2 protein (red) in cultured HCASMC. A number of prominent cells expressing high levels of ACTA2 appeared to have low-level expression of TCF21. Tcf21 anti-sense transcript in situ hybridization was conducted with C) mouse tissue sections and TCF21 hybridizations conducted with D) sections of human coronary artery tissues, revealing high level of specific (blue) labeling of adventitial cells. There was no evidence of staining of the medial or endothelial cell layer. E) Control hybridizations were performed with species relevant sense transcripts. F) Tcf21 $1^{\text {lacZ/t }}$ reporter mice were employed with Xgal in situ staining to investigate $T c f 21$ expression in the adult cardiovascular system. The low power view at left is evaluated with Xgal cytochemical staining (blue) and Acta2 immunostaining (red). The boxed area is localized on the coronary artery and is visualized in panels to the right at high power. $\beta$ galactosidase enzymatic activity was localized primarily to the adventitia, with some Tcf21 expressing cells being located adjacent to the external elastic lamina in juxtaposition to the medial SMC and other cells being localized to the loose adventitial tissue more distantly separated from the vascular wall. Combined immunostaining for Acta2 (red) expression and $\beta$-galactosidase activity (pseudocolored green) did not show colocalization (yellow color) and suggested that Tcf21 expressing cells did not express this SMC marker. G) The low power view at the left shows tissue at the aortic root, evaluated with Xgal cytochemical staining (blue) and Acta2 
immunostaining (red). The boxed area is localized on the aortic wall and is visualized in panels to the right at high power. $T c f 21$ expression visualized as $\beta$-galactosidase activity was observed in proximal aortic medial cells in a patchy distribution, with no apparent overlap in expression for Tcf21 and Acta2 as would be shown with yellow color.

(TIF)

S2 Fig. siTCF21 knockdown for RNA-Seq studies. A) siTCF21 transfected into HCASMC provided a significant decrease in mRNA levels for TCF21, (0.99 \pm 0.02 control vs. $0.25 \pm 0.02$ siTCF21, $P<0.0001)$. B) Western blot of protein extracts from HCASMC that were mock transfected compared to those that were transfected with control siRNA or siTCF21. Quantitation of blots showed a decrease in protein level to $26 \%$ of baseline in the cells treated with siTCF21 compared to siCTRL.

S3 Fig. Gene ontology of the TCF21 Vascular Disease Network derived from RNA-Seq studies of HCASMC exposed to TCF21 knockdown. Differentially regulated genes were employed to construct an interaction network highlighting the gene ontology (GO) annotation information of the network genes. Visualization of the network was performed in Cytoscape. Molecular function gene ontology terms were assigned to the network nodes using the Bingo Cytoscape application and colored with GOlorize Cytoscape. Log values of the relative expression level fold changes are represented in a green-red color palette as a circle surrounding the nodes (red up, green down), unless the gene was not assigned with GO terms in which case fold change is the color of the node. Edges were distinguished as described for Fig 1. (TIF)

S4 Fig. Lentiviral overexpression and shRNA knockdown for in vitro studies in SMC. Control lentiviral vectors (pWPI) and lentiviral overexpression vectors (pWPI-TCF21), and control (pLVTHM) and lentiviral shRNA mediated knockdown vectors (pLVTHM-shTCF21) were used to transduce primary cultured HCASMC. A) pWPI-TCF21 increased TCF21 mRNA levels (1.0 \pm 0.04 pWPI vs. $32.5 \pm 0.02$ pWPI-TCF21, $\mathrm{P}<0.0001)$, and pLVTHM-shTCF21 decreased expression (1.0 \pm 0.06 pLVTHM vs. $0.34 \pm 0.04$ pLVTHM-shTCF21 2, P $<0.001)$. B) Western blots of protein extracts from HCASMC that were transduced with over-expression and knockdown lentiviruses showed a 4.5 -fold increase, and reduction of TCF21 protein levels to $8 \%$

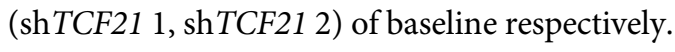
(TIF)

S5 Fig. TCF21 regulates cell division in vitro in HCASMC. A) Flow cytometry of cultured HCASMC transduced with TCF21 overexpressing lentivirus (pWPI-TCF21) or empty lentivirus (pWPI empty), both of which express GFP, was employed to evaluate how TCF21 affects cell division. HCASMC showed an increase in TCF21 overexpressing cells from 48 to 82 percent of the culture within 25 days. B) Similar knockdown experiments were conducted with shRNA expressing lentiviruses (shTCF21 1, shTCF21 2) as well as the parent pLVTHM which served as control. All vectors expressed GFP. There was a significant decrease in GFP positive cells at day 28 , siTCF21 1 vs. siCTRL $P<0.0001$; siTCF21 2 vs. siCTRL $P<0.0001$. Taken together, these data are consistent with a pro-proliferative role for TCF21. (TIF)

S6 Fig. TCF21 and ACTA2 immunohistochemistry of human vascular tissues with varying degrees of disease severity. A-C) In vessels with minimal disease there was adventitial TCF21 staining (red) with some patchy staining of the minimal neointima (red arrows), but no staining in the media which was positive for ACTA2 (brown). D, E) In vessels with significant 
disease there was robust TCF21 staining in the adventitia and neointima and some patchy staining in the fibrous cap, but very little staining in the media. ACTA2 staining was prominent in the fibrous cap and less so in the media. F) Control studies without primary antibodies showed only weak background staining. Lu, lumen; In, neointima; M, media; FC, fibrous cap. (TIF)

S7 Fig. Tcf21 reporter gene expression in mouse vascular tissues with combined immunohistochemical staining for various cellular lineage markers. Various antibodies were employed for lineage markers with tissue from $T c f 21^{\text {lacZ/+ }}, A p o E^{-/-}$animals, Xgal stain is blue and immunohistochemical staining is red for lineage markers.

(TIF)

S8 Fig. Tcf21 expressing cells in $\mathrm{ApoE}^{-/-}$lesions give rise to smooth muscle cells in the fibrous cap. $T c f 21^{i C r e /+}, A_{p o E^{-/-}}$mice were administered tamoxifen to activate expression of an inducible MerCreMer construct knocked into the Tcf21 locus. Cre mediated recombination of a lacZ reporter at the constitutively expressed ROSA26 locus allowed lineage tracing of Tcf 21 expressing cells. Animals received tamoxifen at 6-8 weeks of HFD and tissues were harvested at 12 weeks of diet. A region of atheroma identified by the box in the low power image on the left and shown in high power fields in images on the right show blue $\beta$-galactosidase positive Tcf21 lineage traced cells forming a subcapsular structure. These cells were not labeled by immunostaining for Tagln (red). Similar $\beta$-galactosidase positive Tcf21 lineage traced cells were also seen in association with the fibrous cap and these cells did stain positive for Tagln expression.

S1 Table. Cardiovascular disease functional annotation categories derived from siTCF21 knockdown and RNA-Seq studies of differentially expressed genes. (XLSX)

S2 Table. Cardiovascular system development and function annotation categories derived from siTCF21 knockdown and RNA-Seq studies of differentially expressed genes. (XLSX)

S3 Table. Cellular and molecular function terms enriched in "vascular disease" category identified with IPA analysis of siTCF21 knockdown and RNA-Seq studies of differentially expressed genes.

(XLSX)

\section{Acknowledgments}

The authors thank Eric Olson and Rhonda Bassel-Duby, University of Texas Southwestern, for $T c f 21^{\text {lac } Z}$ and $T c f 21^{i C r e}$ mice, and Muredach Reilly, University of Pennsylvania, for providing human coronary artery samples.

\section{Author Contributions}

Conceived and designed the experiments: STN WAB GKO MDT TQ. Performed the experiments: STN KC AR CLM KA RK ICO YX NT NM LP SA VN. Analyzed the data: STN TQ JBK MP. Contributed reagents/materials/analysis tools: WAB GKO MDT. Wrote the paper: MDT TQ JBK UH LM. 


\section{References}

1. Daviglus ML, Talavera GA, Aviles-Santa ML, Allison M, Cai J, et al. (2012) Prevalence of major cardiovascular risk factors and cardiovascular diseases among Hispanic/Latino individuals of diverse backgrounds in the United States. JAMA: the journal of the American Medical Association 308: 1775-1784. doi: 10.1001/jama.2012.14517 PMID: 23117778

2. Gupta R, Gupta KD (2009) Coronary heart disease in low socioeconomic status subjects in India: "an evolving epidemic". Indian heart journal 61: 358-367. PMID: 20635739

3. Ikem I, Sumpio BE (2011) Cardiovascular disease: the new epidemic in sub-Saharan Africa. Vascular 19: 301-307. doi: 10.1258/vasc.2011.ra0049 PMID: 21940758

4. Almahmeed W, Arnaout MS, Chettaoui R, Ibrahim M, Kurdi MI, et al. (2012) Coronary artery disease in Africa and the Middle East. Therapeutics and clinical risk management 8: 65-72. doi: 10.2147/TCRM. S26414 PMID: 22368447

5. Gomez D, Owens GK (2012) Smooth muscle cell phenotypic switching in atherosclerosis. Cardiovasc Res 95: 156-164. doi: 10.1093/cvr/cvs115 PMID: 22406749

6. Deloukas P, Kanoni S, Willenborg C, Farrall M, Assimes TL, et al. (2012) Large-scale association analysis identifies new risk loci for coronary artery disease. Nat Genet 45: 25-33. doi: 10.1038/ng.2480 PMID: 23202125

7. Lu X, Wang L, Chen S, He L, Yang X, et al. (2012) Genome-wide association study in Han Chinese identifies four new susceptibility loci for coronary artery disease. Nat Genet 44: 890-894. doi: 10.1038/ ng.2337 PMID: 22751097

8. Schunkert H, Konig IR, Kathiresan S, Reilly MP, Assimes TL, et al. (2011) Large-scale association analysis identifies 13 new susceptibility loci for coronary artery disease. Nat Genet 43: 333-338. doi: 10.1038/ng.784 PMID: 21378990

9. Cui S, Ross A, Stallings N, Parker KL, Capel B, et al. (2004) Disrupted gonadogenesis and male-tofemale sex reversal in Pod1 knockout mice. Development 131: 4095-4105. PMID: 15289436

10. Cui S, Schwartz L, Quaggin SE (2003) Pod1 is required in stromal cells for glomerulogenesis. Dev Dyn 226: 512-522. PMID: 12619136

11. Hidai H, Bardales R, Goodwin R, Quertermous T, Quertermous EE (1998) Cloning of capsulin, a basic helix-loop-helix factor expressed in progenitor cells of the pericardium and the coronary arteries. Mech Dev 73: 33-43. PMID: 9545526

12. Lu J, Chang P, Richardson JA, Gan L, Weiler H, et al. (2000) The basic helix-loop-helix transcription factor capsulin controls spleen organogenesis. Proc Natl Acad Sci U S A 97: 9525-9530. PMID: 10944221

13. Lu J, Richardson JA, Olson EN (1998) Capsulin: a novel bHLH transcription factor expressed in epicardial progenitors and mesenchyme of visceral organs. Mech Dev 73: 23-32. PMID: 9545521

14. Lu JR, Bassel-Duby R, Hawkins A, Chang P, Valdez R, et al. (2002) Control of facial muscle development by MyoR and capsulin. Science 298: 2378-2381. PMID: 12493912

15. Quaggin SE, Schwartz L, Cui S, Igarashi P, Deimling J, et al. (1999) The basic-helix-loop-helix protein pod1 is critically important for kidney and lung organogenesis. Development 126: 5771-5783. PMID: 10572052

16. Robb L, Mifsud L, Hartley L, Biben C, Copeland NG, et al. (1998) epicardin: A novel basic helix-loophelix transcription factor gene expressed in epicardium, branchial arch myoblasts, and mesenchyme of developing lung, gut, kidney, and gonads. Dev Dyn 213: 105-113. PMID: 9733105

17. Acharya A, Baek ST, Huang G, Eskiocak B, Goetsch S, et al. (2012) The bHLH transcription factor Tcf21 is required for lineage-specific EMT of cardiac fibroblast progenitors. Development 139: 2139-2149. doi: 10.1242/dev.079970 PMID: 22573622

18. Braitsch CM, Combs MD, Quaggin SE, Yutzey KE (2012) Pod1/Tcf21 is regulated by retinoic acid signaling and inhibits differentiation of epicardium-derived cells into smooth muscle in the developing heart. Dev Biol 368: 345-357. doi: 10.1016/j.ydbio.2012.06.002 PMID: 22687751

19. Feng J, Mantesso A, Sharpe PT (2010) Perivascular cells as mesenchymal stem cells. Expert opinion on biological therapy 10: 1441-1451. doi: 10.1517/14712598.2010.517191 PMID: 20836622

20. Sheikh AY, Chun HJ, Glassford AJ, Kundu RK, Kutschka I, et al. (2008) In vivo genetic profiling and cellular localization of apelin reveals a hypoxia-sensitive, endothelial-centered pathway activated in ischemic heart failure. Am J Physiol Heart Circ Physiol 294: H88-98. PMID: 17906101

21. Anders S, Huber W (2010) Differential expression analysis for sequence count data. Genome Biol 11: R106. doi: 10.1186/gb-2010-11-10-r106 PMID: 20979621 
22. Robinson MD, McCarthy DJ, Smyth GK (2010) edgeR: a Bioconductor package for differential expression analysis of digital gene expression data. Bioinformatics 26: 139-140. doi: 10.1093/bioinformatics/ btp616 PMID: 19910308

23. Soneson C, Delorenzi M (2013) A comparison of methods for differential expression analysis of RNAseq data. BMC Bioinformatics 14: 91. doi: 10.1186/1471-2105-14-91 PMID: 23497356

24. Mack CP, Owens GK (1999) Regulation of smooth muscle alpha-actin expression in vivo is dependent on CArG elements within the $5^{\prime}$ and first intron promoter regions. Circ Res 84: 852-861. PMID: 10205154

25. Arab K, Smith LT, Gast A, Weichenhan D, Huang JP, et al. (2011) Epigenetic deregulation of TCF21 inhibits metastasis suppressor KISS1 in metastatic melanoma. Carcinogenesis 32: 1467-1473. doi: 10. 1093/carcin/bgr138 PMID: 21771727

26. Barnes RM, Firulli AB (2009) A twist of insight-the role of Twist-family bHLH factors in development. Int J Dev Biol 53: 909-924. doi: 10.1387/ijdb.082747rb PMID: 19378251

27. Li W, Li Q, Jiao Y, Qin L, Ali R, et al. (2014) Tgfbr2 disruption in postnatal smooth muscle impairs aortic wall homeostasis. J Clin Invest 124: 755-767. doi: 10.1172/JCI69942 PMID: 24401272

28. Inamoto S, Kwartler CS, Lafont AL, Liang YY, Fadulu VT, et al. (2010) TGFBR2 mutations alter smooth muscle cell phenotype and predispose to thoracic aortic aneurysms and dissections. Cardiovasc Res 88: 520-529. doi: 10.1093/cvr/cvq230 PMID: 20628007

29. Mellgren AM, Smith CL, Olsen GS, Eskiocak B, Zhou B, et al. (2008) Platelet-derived growth factor receptor beta signaling is required for efficient epicardial cell migration and development of two distinct coronary vascular smooth muscle cell populations. Circ Res 103: 1393-1401. doi: 10.1161/ CIRCRESAHA.108.176768 PMID: 18948621

30. Zhang Y, Riesterer C, Ayrall AM, Sablitzky F, Littlewood TD, et al. (1996) Inducible site-directed recombination in mouse embryonic stem cells. Nucleic Acids Res 24: 543-548. PMID: 8604292

31. Acharya A, Baek ST, Banfi S, Eskiocak B, Tallquist MD (2011) Efficient inducible Cre-mediated recombination in Tcf21 cell lineages in the heart and kidney. Genesis 49: 870-877. doi: 10.1002/dvg.20750 PMID: 21432986

32. Alexander MR, Owens GK (2012) Epigenetic control of smooth muscle cell differentiation and phenotypic switching in vascular development and disease. Annu Rev Physiol 74: 13-40. doi: 10.1146/ annurev-physiol-012110-142315 PMID: 22017177

33. Owens GK, Kumar MS, Wamhoff BR (2004) Molecular regulation of vascular smooth muscle cell differentiation in development and disease. Physiol Rev 84: 767-801. PMID: 15269336

34. Funato N, Ohyama K, Kuroda T, Nakamura M (2003) Basic helix-loop-helix transcription factor epicardin/capsulin/Pod-1 suppresses differentiation by negative regulation of transcription. J Biol Chem 278: 7486-7493. PMID: 12493738

35. Orlandi A, Bennett M (2010) Progenitor cell-derived smooth muscle cells in vascular disease. Biochemical pharmacology 79: 1706-1713. doi: 10.1016/j.bcp.2010.01.027 PMID: 20117099

36. Frid MG, Aldashev AA, Nemenoff RA, Higashito R, Westcott JY, et al. (1999) Subendothelial cells from normal bovine arteries exhibit autonomous growth and constitutively activated intracellular signaling. Arterioscler Thromb Vasc Biol 19: 2884-2893. PMID: 10591665

37. Holifield B, Helgason T, Jemelka S, Taylor A, Navran S, et al. (1996) Differentiated vascular myocytes: are they involved in neointimal formation? J Clin Invest 97: 814-825. PMID: 8609239

38. Sainz J, Al Haj Zen A, Caligiuri G, Demerens C, Urbain D, et al. (2006) Isolation of "side population" progenitor cells from healthy arteries of adult mice. Arterioscler Thromb Vasc Biol 26: 281-286. PMID: 16306431

39. Majesky MW, Dong XR, Hoglund V, Mahoney WM Jr., Daum G (2011) The adventitia: a dynamic interface containing resident progenitor cells. Arterioscler Thromb Vasc Biol 31: 1530-1539. doi: 10.1161/ ATVBAHA.110.221549 PMID: 21677296

40. Zengin E, Chalajour F, Gehling UM, Ito WD, Treede H, et al. (2006) Vascular wall resident progenitor cells: a source for postnatal vasculogenesis. Development 133: 1543-1551. PMID: 16524930

41. Virmani R, Burke AP, Farb A, Kolodgie FD (2006) Pathology of the vulnerable plaque. J Am Coll Cardiol 47: C13-18. PMID: 16631505

42. Kozaki K, Kaminski WE, Tang J, Hollenbach S, Lindahl P, et al. (2002) Blockade of platelet-derived growth factor or its receptors transiently delays but does not prevent fibrous cap formation in ApoE null mice. Am J Pathol 161: 1395-1407. PMID: 12368212

43. Sano H, Sudo T, Yokode M, Murayama T, Kataoka H, et al. (2001) Functional blockade of plateletderived growth factor receptor-beta but not of receptor-alpha prevents vascular smooth muscle cell accumulation in fibrous cap lesions in apolipoprotein E-deficient mice. Circulation 103: 2955-2960. PMID: 11413086 
44. Li G, Jin R, Norris RA, Zhang L, Yu S, et al. (2010) Periostin mediates vascular smooth muscle cell migration through the integrins alphavbeta3 and alphavbeta5 and focal adhesion kinase (FAK) pathway. Atherosclerosis 208: 358-365. doi: 10.1016/j.atherosclerosis.2009.07.046 PMID: 19695571

45. Litvin J, Chen X, Keleman S, Zhu S, Autieri M (2007) Expression and function of periostin-like factor in vascular smooth muscle cells. Am J Physiol Cell Physiol 292: C1672-1680. PMID: 17092992

46. Lindner V, Wang Q, Conley BA, Friesel RE, Vary CP (2005) Vascular injury induces expression of periostin: implications for vascular cell differentiation and migration. Arterioscler Thromb Vasc Biol 25: 77-83. PMID: 15514205

47. Hixson JE, Shimmin LC, Montasser ME, Kim DK, Zhong Y, et al. (2011) Common variants in the periostin gene influence development of atherosclerosis in young persons. Arterioscler Thromb Vasc Biol 31: 1661-1667. doi: 10.1161/ATVBAHA.111.224352 PMID: 21474826

48. Bentzon JF, Falk E (2010) Circulating smooth muscle progenitor cells in atherosclerosis and plaque rupture: current perspective and methods of analysis. Vascular pharmacology 52: 11-20. doi: 10.1016/ j.vph.2009.11.005 PMID: 19951745

49. Yu H, Stoneman V, Clarke M, Figg N, Xin HB, et al. (2011) Bone marrow-derived smooth muscle-like cells are infrequent in advanced primary atherosclerotic plaques but promote atherosclerosis. Arterioscler Thromb Vasc Biol 31: 1291-1299. doi: 10.1161/ATVBAHA.110.218578 PMID: 21372299

50. Miller CL, Anderson DR, Kundu RK, Raiesdana A, Nurnberg ST, et al. (2013) Disease-Related Growth Factor and Embryonic Signaling Pathways Modulate an Enhancer of TCF21 Expression at the 6q23.2 Coronary Heart Disease Locus. PLoS Genet 9: e1003652. doi: 10.1371/journal.pgen.1003652 PMID: 23874238

51. Miller CL, Haas U, Diaz R, Leeper NJ, Kundu RK, et al. (2014) Coronary Heart Disease-Associated Variation in TCF21 Disrupts a miR-224 Binding Site and miRNA-Mediated Regulation. PLoS Genet 10: e1004263. doi: 10.1371/journal.pgen.1004263 PMID: 24676100

52. Chen YC, Bui AV, Diesch J, Manasseh R, Hausding C, et al. (2013) A novel mouse model of atherosclerotic plaque instability for drug testing and mechanistic/therapeutic discoveries using gene and microRNA expression profiling. Circ Res 113: 252-265. doi: 10.1161/CIRCRESAHA.113.301562 PMID: 23748430

53. Aono J, Suzuki J, Iwai M, Horiuchi M, Nagai T, et al. (2012) Deletion of the angiotensin II type 1a receptor prevents atherosclerotic plaque rupture in apolipoprotein E-/- mice. Arterioscler Thromb Vasc Biol 32: 1453-1459. doi: 10.1161/ATVBAHA.112.249516 PMID: 22460554

54. Hidai C, Zupancic T, Penta K, Mikhail A, Kawana M, et al. (1998) Cloning and characterization of developmental endothelial locus-1: an embryonic endothelial cell protein that binds the alphavbeta3 integrin receptor. Genes Dev 12: 21-33. PMID: 9420328

55. Perisic L, Hedin E, Razuvaev A, Lengquist M, Osterholm C, et al. (2013) Profiling of atherosclerotic lesions by gene and tissue microarrays reveals PCSK 6 as a novel protease in unstable carotid atherosclerosis. Arterioscler Thromb Vasc Biol 33: 2432-2443. doi: 10.1161/ATVBAHA.113.301743 PMID: 23908247 